\title{
Letter
}

\section{DNA microarray analysis of fetal liver of C57BL/6J mice exposed to cadmium during gestation}

\author{
Hisaka Kurita' ${ }^{1}$, Hisamitsu Nagase ${ }^{1}$, Maki Tokumoto ${ }^{2}$, Jin-Yong Lee ${ }^{2}$ and Masahiko Satoh ${ }^{2}$ \\ 'Laboratory of Hygienic Chemistry and Molecular Toxicology, Gifu Pharmaceutical University, \\ 1-25-4 Daigaku-nishi, Gifu 501-1196, Japan \\ ${ }^{2}$ Laboratory of Pharmaceutical Health Sciences, School of Pharmacy, Aichi Gakuin University, \\ 1-100 Kusumoto-cho, Chikusa-ku, Nagoya 464-8650, Japan
}

(Received November 15, 2016; Accepted November 15, 2016)

\begin{abstract}
Cadmium (Cd) is a non-essential toxic metal widely distributed throughout the environment. $\mathrm{Cd}$ is reported to be toxic to the fetus, so we aimed to investigate changes in gene expression in the liver of fetal mice exposed to Cd during gestation. We exposed pregnant mice to $\mathrm{Cd}(5 \mathrm{mg} / \mathrm{kg})$ and collected fetal livers to perform DNA microarray analysis. The expression of 1,669 genes was found to be increased more than 2.0-fold, while that of 194 genes was decreased less than 0.5 -fold in fetal livers following $\mathrm{Cd}$ exposure during gestation. We categorized the Cd-changed genes in terms of cell cycle and cell proliferation, apoptosis, cell growth and differentiation, cellular defense, metabolism, transport, transcription, signal transduction, metal homeostasis, and ubiquitin proteasome system. These results provide useful information about fetal toxicity following gestational $\mathrm{Cd}$ exposure.
\end{abstract}

Key words: Cadmium, Gestational exposure, Fetal toxicity, DNA microarray

\section{INTRODUCTION}

Cadmium $(\mathrm{Cd})$ is a non-essential toxic metal widely found throughout the environment. Humans are typically exposed to $\mathrm{Cd}$ on a daily basis from foodstuffs or tobacco, and such exposure has been shown to cause toxic effects in the liver, kidney, lung, bone, and reproductive tissues (Satoh et al., 2002). The risk for Cd toxicity is elevated during pregnancy. Previous epidemiological studies demonstrated that the maternal body burden of $\mathrm{Cd}$ is associated with low birth weight (Nishijo et al., 2004) and low birth height (Nishijo et al., 2002), while maternal $\mathrm{Cd}$ exposure during animal gestation is related to fetal death (Levin and Miller, 1980).

Despite these associations, the genes involved in $\mathrm{Cd}-$ induced fetal toxicity following gestational exposure have not been identified. Therefore, in the present study, we performed DNA microarray analysis of mouse fetal livers exposed to $\mathrm{Cd}$ during gestation to screen for the genes responsible for $\mathrm{Cd}$ fetal toxicity.

\section{MATERIALS AND METHODS}

\section{Animal experiments}

C57BL/6J mice were a generous gift from Dr. C. Tohyama (University of Tokyo, Japan). Animals were maintained at a controlled temperature and humidity with $12 \mathrm{hr} / 12 \mathrm{hr}$ light/dark cycles, and given free access to rodent chow and water. All experiments were performed in accordance with the Guidelines for Animal Experiments of Gifu Pharmaceutical University. Nulliparous female mice aged 8 weeks were housed with one male of the same strain for $24 \mathrm{hr}$. After the mating period, females were separated from the males and housed individually in plastic cages from gestational day 1 (GD1). Dams were split into two groups: those receiving $5 \mathrm{mg} \mathrm{Cd} / \mathrm{kg}$ $(n=5)$ and the control group $(n=4)$. Cd solution was prepared with $\mathrm{CdCl}_{2}$ (2.5-hydrous powder, 99.9\% purification; Wako Pure Chemical Industries, Osaka, Japan) in distillated water. A single dose of Cd solution was orally administrated daily to the pregnant mice from GD1 to GD18. The control group received distilled water for the same period by oral administration. On GD19, pregnant mice were deeply anesthetized with ether and fetal livers were removed. Fetal livers were pooled for each litter and

Correspondence: Masahiko Satoh (E-mail: masahiko@dpc.agu.ac.jp) 
samples were quick-frozen and stored at $-80^{\circ} \mathrm{C}$.

\section{Extraction of total RNA}

Total RNA was isolated from pooled fetal livers using the SV total RNA isolation system (Promega, Madison, WI, USA) according to the manufacturer's protocol.

\section{DNA microarray analysis}

Pooled total RNA $(100 \mu \mathrm{g})$ was reverse transcribed into cDNA by Superscript II reverse transcriptase (Invitrogen, Grand Island, NY, USA) using Oligo (dT)1218 primer (Invitrogen) and dNTP mix (Invitrogen) containing aminoallyl-dUTP (Ambion, Austin, TX, USA) in accordance with the manufacturer's instructions. cDNA from control and Cd-treated groups was labeled with fluorescent dyes Cy3 and Cy5 (Amersham Bioscience, Little Chalfont, UK), respectively. The mixture of labeled cDNA from control and Cd-treated groups was hybridized with an AceGene ${ }^{\circledR}$ Oligo Chip 30K (Hitachi Software, Kanagawa, Japan) using a Lucidea SlidePro (Amersham Bioscience). Oligonucleotide-based microarray slides used for the target gene search contained approximately 30,000 spots of mouse DNA. After hybridization, the slides were washed and scanned by the $\mathrm{CRBIO}^{\mathrm{TM}} \mathrm{II}$ (Hitachi Software). The fluorescent intensity of the spots was measured for $\mathrm{Cy} 3$ and $\mathrm{Cy} 5$, and the induction ratio of each gene was calculated with DNASIS ${ }^{\circledR}$ Array, version 2.4 software (Hitachi Software). Information about each gene on the microarray slide was obtained from the NCBI database. Genes showing expression changes were categorized according to function by referring to the Gene Ontology database.

\section{RESULTS AND DISCUSSION}

We found that the expression of 1,669 genes was increased more than 2.0-fold, and the expression of 194 genes was decreased less than 0.5 -fold in the fetal mouse liver following $\mathrm{Cd}$ exposure during gestation. The altered genes were categorized according to function by referring to the Gene Ontology database. The expression of 69 genes associated with the cell cycle and cell proliferation was increased, and that of five genes was decreased (Tables 1, 2). The expression of 49 genes related to apoptosis was increased, and that of eight genes was decreased (Tables 3, 4). The expression of 57 genes associated with cell growth and differentiation was increased, while that of nine genes was decreased (Tables 5, 6). The expression of 69 genes related to cellular defense was increased, compared with five genes that decreased in expression (Tables 7,8 ). Ninety-two genes related to metabolism demonstrated increased expression, while 12 showed decreased expression (Tables 9, 10). Increased expression of 244 genes related to transport was observed, as well as decreased expression of 21 such genes (Tables $11,12)$. The expression of 162 genes related to transcription was increased, and that of 19 genes was decreased (Tables 13, 14). Changes in signal transduction-associated genes were reflected by increased expression in 144 and decreased expression in 32 (Tables 15, 16). The expression of 34 genes related to metal homeostasis, including $M t 1$, was increased, while three such genes showed decreased expression (Tables 17, 18). Thirty-two genes related to the ubiquitin proteasome system showed increased expression, compared with three such genes that were decreased (Tables 19, 20). Other genes in the fetal liver changed by gestational $\mathrm{Cd}$ exposure are shown in Tables 21 and 22.

Amotl2 expression was found to be increased in the fetal liver of mice exposed to $\mathrm{Cd}$ during gestation (Table 21), which supports our earlier study that demonstrated higher AMOTL2 expression in HK-2 human proximal tubular cells treated with Cd compared with untreated cells (Lee et al., 2013). The present study also observed increased expression of Cdkn2b (Table 1), Pvrl3 (Table 21), and $U g d h$ (Table 11), and decreased expression of Ptpru (Table 22); the same changes were previously observed in the kidneys of mice exposed to $\mathrm{Cd}$ for 12 months (Tokumoto et al., 2013a). Similarly, we observed increased expression of Srpr (Table 11), Wiz (Table 17), Jub (Table 21), Taldo1 (Table 9), Oat (Table 21), and Ctbpl (Table 9), and decreased expression of Tcf20 (Table 14), which was previously seen in NRK-52E rat kidney epithelial cells treated with Cd (Tokumoto et al., 2011). The livers of mice exposed to $\mathrm{Cd}$ for 30 days were previously reported to have increased Rpa 2 expression and decreased Tcf20 expression (Tokumoto et al., 2013b), and our current findings support these results (Tables 14 and 21).

Therefore, our present study identified several common genes that might be responsible for $\mathrm{Cd}$ fetal toxicity and that are involved in similar hepatic and renal toxicity pathways. These findings provide useful information for understanding $\mathrm{Cd}$-induced gene alteration in the fetus.

\section{ACKNOWLEDGMENTS}

This study was supported by Study of the Health Effects of Heavy Metals Organized by the Ministry of Environment, Japan.

Conflict of interest---- The authors declare that there is no conflict of interest. 
Gene expression changes in fetal liver of gestational Cd-exposed mice

Table 1. Up-regulated genes related to cell cycle and cell proliferation in the mouse fetal liver following gestational $\mathrm{Cd}$ exposure.

\begin{tabular}{|c|c|c|c|c|c|}
\hline Accession Number & Gene Name & Fold of change & Accession Number & Gene Name & Fold of change \\
\hline NM_021399 & $B c l 11 b$ & 3.42 & NM_138630 & Arhgap4 & 2.34 \\
\hline NM_011835 & Katnal & 3.02 & NM_009627 & $A d m$ & 2.33 \\
\hline NM_023233 & Trim13 & 2.96 & NM_010798 & Mif & 2.33 \\
\hline NM_011806 & Dmtf1 & 2.93 & NM_007950 & Ereg & 2.32 \\
\hline NM_009353 & $\operatorname{Terf} 2$ & 2.87 & NM_009828 & Ccna2 & 2.30 \\
\hline NM_027985 & $\operatorname{Mad} 2 \mathrm{l} 2$ & 2.86 & NM_009791 & Calmbpl & 2.29 \\
\hline NM_028228 & Pinxl & 2.85 & NM_011379 & Sipal & 2.28 \\
\hline NM_009368 & $\operatorname{Tg} f b 3$ & 2.83 & NM_017464 & Nedd9 & 2.28 \\
\hline NM_009352 & Terf1 & 2.78 & NM_016964 & Stag3 & 2.26 \\
\hline NM_010690 & Lats1 & 2.75 & NM_007783 & Csk & 2.25 \\
\hline NM_023326 & Bmyc & 2.74 & NM_007836 & Gadd45a & 2.25 \\
\hline NM_009465 & $A x l$ & 2.70 & NM_134092 & $M t b p$ & 2.24 \\
\hline NM_009379 & Thpo & 2.67 & AF179996 & 8-Sep & 2.21 \\
\hline NM_011200 & Ptp4al & 2.66 & NM_007671 & $C d k n 2 c$ & 2.20 \\
\hline NM_011408 & Slfn2 & 2.65 & NM_009772 & Bubl & 2.19 \\
\hline NM_016692 & Incenp & 2.58 & NM_010509 & Ifnar2 & 2.19 \\
\hline NM_016746 & Ccnc & 2.55 & NM_009050 & Ret & 2.18 \\
\hline NM_021328 & $\operatorname{Bin} 3$ & 2.55 & NM_009875 & $C d k n 1 b$ & 2.18 \\
\hline NM_010208 & $\mathrm{Fgr}$ & 2.52 & NM_011487 & Stat4 & 2.16 \\
\hline NM_001081058 & $C d c 2 l 5$ & 2.50 & NM_008583 & Men1 & 2.16 \\
\hline NM_009506 & $\operatorname{Veg} f c$ & 2.49 & NM_011237 & $\operatorname{Rad} 9$ & 2.16 \\
\hline NM_008059 & $G 0 s 2$ & 2.48 & NM_011647 & $T s c 2$ & 2.14 \\
\hline NM_011462 & Spin & 2.47 & NM_015771 & Lats2 & 2.13 \\
\hline NM_018830 & Asah2 & 2.46 & NM_022889 & Pes 1 & 2.13 \\
\hline NM_009516 & Weel & 2.46 & NM_010716 & Lig3 & 2.12 \\
\hline NM_027764 & Rcbtbl & 2.44 & NM_010757 & Mafk & 2.11 \\
\hline NM_020572 & Aurkc & 2.43 & NM_010204 & $F g f 6$ & 2.10 \\
\hline NM_009625 & Adcyapl & 2.42 & NM_013538 & $\mathrm{Cdca} 3$ & 2.10 \\
\hline NM_130860 & $C d k 9$ & 2.40 & NM_011601 & $\mathrm{Tlm}$ & 2.10 \\
\hline NM_011516 & Sycpl & 2.39 & NM_025415 & $C k s 2$ & 2.07 \\
\hline NM_021515 & $A k 1$ & 2.39 & NM_138585 & Cherp & 2.06 \\
\hline NM_017461 & $1-S e p$ & 2.38 & NM_021409 & Pard6b & 2.05 \\
\hline NM_013636 & Ppplcc & 2.37 & NM_007670 & $C d k n 2 b$ & 2.04 \\
\hline NM_007934 & Enpep & 2.36 & NM_023284 & Cdcal & 2.01 \\
\hline NM 009974 & Csnk2a2 & 2.35 & & & \\
\hline
\end{tabular}

Table 2. Down-regulated genes related to cell cycle and cell proliferation in the mouse fetal liver following gestational $\mathrm{Cd}$ exposure.

\begin{tabular}{llcllc}
\hline Accession Number & Gene Name & Fold of change & Accession Number & Gene Name & Fold of change \\
\hline NM_016904 & Cks1 & 0.417 & NM_153599 & Cdk8 & 0.453 \\
NM_145851 & Cables2 & 0.438 & NM_175660 & Hist1h2ab & 0.460 \\
NM_013625 & Pafahlb1 & 0.450 & & &
\end{tabular}


H. Kurita et al.

Table 3. Up-regulated genes related to apoptosis in the mouse fetal liver following gestational Cd exposure.

\begin{tabular}{llllll}
\hline Accession Number & Gene Name & Fold of change & Accession Number & Gene Name & Fold of change \\
\hline NM_013542 & Gzmb & 3.13 & NM_001033126 & Tnfrsf7 & 2.31 \\
AF353993 & Bbp & 3.08 & NM_016802 & Arha & 2.30 \\
NM_009688 & Birc4 & 2.87 & NM_008234 & Hells & 2.30 \\
NM_009163 & Sgpl1 & 2.84 & NM_007465 & Birc3 & 2.26 \\
NM_153552 & Thoc1 & 2.78 & NM_007466 & Api5 & 2.25 \\
NM_007609 & Casp4 & 2.77 & NM_023566 & Muc2 & 2.24 \\
NM_030750 & Sgpp1 & 2.76 & NM_009383 & Tiall & 2.22 \\
NM_009472 & Unc5c & 2.72 & NM_007828 & Dapk3 & 2.22 \\
NM_146001 & Hip1 & 2.71 & NM_007829 & Daxx & 2.21 \\
NM_010100 & Edar & 2.69 & NM_008798 & Pdcd1 & 2.20 \\
NM_007669 & Cdknla & 2.62 & NM_009684 & Apaf1 & 2.20 \\
NM_028133 & Egln3 & 2.58 & NM_025778 & Bcl2l14 & 2.15 \\
NM_010202 & Fgf4 & NM_009068 & Ripk1 & 2.14 \\
NM_010164 & Eya1 & 2.55 & NM_019745 & Pdcd10 & 2.11 \\
NM_016688 & Pdcd7 & 2.53 & NM_007891 & E2f1 & 2.10 \\
NM_011632 & Traf3 & 2.50 & NM_133821 & Plekhe1 & 2.08 \\
NM_019955 & Ripk3 & 2.50 & NM_007544 & Bid & 2.08 \\
NM_010431 & Hifla & 2.47 & NM_007955 & Ptprv & 2.08 \\
NM_009736 & Bag1 & 2.46 & NM_023565 & Csell & 2.07 \\
NM_009367 & Tgfb2 & 2.44 & NM_028283 & Uaca & 2.07 \\
NM_020024 & Taf10 & 2.44 & NM_019464 & Sh3glb1 & 2.07 \\
NM_025680 & Ctnnbl1 & 2.40 & NM_009071 & Rock1 & 2.05 \\
NM_010830 & Msh6 & 2.39 & NM_007870 & Dnase1l3 & 2.02 \\
NM_008714 & Notch1 & 2.37 & NM_011641 & Trp63 & 2.00 \\
NM_010813 & Mnt & 2.36 & & & \\
\hline & & 2.34 & & & \\
\end{tabular}

Table 4. Down-regulated genes related to apoptosis in the mouse fetal liver following gestational Cd exposure.

\begin{tabular}{llcllc}
\hline Accession Number & Gene Name & Fold of change & Accession Number & Gene Name & Fold of change \\
\hline NM_020272 & Pik3cg & 0.244 & NM_020275 & Tnfrsf10b & 0.472 \\
NM_007523 & Bak1 & 0.363 & NM_029653 & Dapk1 & 0.475 \\
NM_023229 & Fastk & 0.400 & NM_009743 & Bcl2l & 0.479 \\
NM_175093 & Ifld & 0.459 & AF054611 & Mapk8ip & 0.485 \\
\hline
\end{tabular}

Table 5. Up-regulated genes related to cell growth, differentiation, and development in the mouse fetal liver following gestational Cd exposure.

\begin{tabular}{llcllc}
\hline Accession Number & Gene Name & Fold of change & Accession Number & Gene Name & Fold of change \\
\hline NM_013865 & Ndr3 & 3.28 & NM_015748 & Slit1 & 2.34 \\
NM_016845 & Acrbp & 2.97 & NM_001170561 & Sbfl & 2.33 \\
NM_015800 & Crim1 & 2.95 & NM_028296 & Car10 & 2.30 \\
NM_007558 & Bmp8a & 2.90 & NM_009574 & Zic2 & 2.30 \\
NM_008871 & Serpine1 & 2.87 & NM_028889 & Efhd1 & 2.27 \\
NM_009469 & Ulk1 & 2.85 & NM_011976 & Sema4g & 2.27 \\
NM_019569 & Fscn3 & 2.81 & NM_011385 & Ski & 2.24 \\
NM_021351 & Cryba4 & 2.81 & NM_030699 & Ntng1 & 2.23 \\
NM_010142 & Ephb2 & 2.80 & NM_018865 & Wisp1 & 2.22 \\
NM_008994 & Pxmp3 & 2.78 & NM_019685 & Ruvbl1 & 2.20 \\
\hline
\end{tabular}


Gene expression changes in fetal liver of gestational Cd-exposed mice

Table 5. (Continued).

\begin{tabular}{llcllc}
\hline Accession Number & Gene Name & Fold of change & Accession Number & Gene Name & Fold of change \\
\hline NM_016701 & Nes & 2.77 & NM_011991 & Cops3 & 2.19 \\
NM_020604 & Jph1 & 2.73 & NM_009640 & Agpt & 2.18 \\
NM_024291 & Ky & 2.72 & NM_026131 & Pdlim7 & 2.13 \\
NM_011349 & Sema3f & 2.71 & NM_023653 & Wnt2 & 2.12 \\
NM_019564 & Prss11 & 2.65 & NM_033565 & Laf4l & 2.11 \\
NM_011248 & Robo3 & 2.58 & NM_016697 & Gpc3 & 2.11 \\
NM_172493 & Diap2 & 2.55 & NM_032002 & Nrg4 & 2.10 \\
NM_013658 & Sema4a & 2.53 & NM_021383 & Rqcd1 & 2.09 \\
NM_013564 & Insl3 & 2.50 & NM_009396 & Tnfaip2 & 2.09 \\
NM_007548 & Prdm1 & 2.49 & NM_008206 & H2-Oa & 2.09 \\
NM_009153 & Sema3b & 2.48 & NM_026570 & Gas41 & 2.08 \\
NM_008742 & Ntf3 & 2.47 & NM_010563 & Ina & 2.08 \\
NM_007553 & Bmp2 & 2.44 & NM_009388 & Tkt & 2.04 \\
NM_009228 & Snta1 & NM_023317 & Nde1 & 2.04 \\
NM_027497 & Epc1 & 2.41 & NM_019754 & Tagln3 & 2.03 \\
NM_024431 & Morf4l1 & 2.39 & NM_007500 & Atoh1 & 2.02 \\
NM_010513 & Igflr & 2.38 & NM_013471 & Anxa4 & 2.01 \\
NM_010516 & Cyr61 & 2.38 & NM_011652 & Ttn & 2.01 \\
NM_010950 & Numbl & 2.38 & & &
\end{tabular}

Table 6. Down-regulated genes related to cell growth, differentiation, and development in the mouse fetal liver following gestational $\mathrm{Cd}$ exposure.

\begin{tabular}{llcllc}
\hline Accession Number & Gene Name & Fold of change & Accession Number & Gene Name & Fold of change \\
\hline NM_144761 & Crygb & 0.239 & NM_008520 & Ltbp3 & 0.455 \\
NM_145506 & Epb4.1l5 & 0.330 & NM_010111 & Efnb2 & 0.464 \\
NM_010279 & Gfra1 & 0.436 & NM_008342 & Igfbp2 & 0.465 \\
NM_010518 & Igfbp5 & 0.446 & NM_008398 & Itga7 & 0.500 \\
NM_013637 & Prm1 & 0.450 & & & \\
\hline
\end{tabular}

Table 7. Up-regulated genes related to cellular defense in the mouse fetal liver following gestational Cd exposure.

\begin{tabular}{llcllc}
\hline Accession Number & Gene Name & Fold of change & Accession Number & Gene Name & Fold of change \\
\hline NM_030677 & Gpx2 & 3.42 & NM_010260 & Gbp2 & 2.36 \\
NM_011329 & Ccl1 & 3.39 & NM_030612 & AA408868 & 2.35 \\
NM_008533 & Ly78 & 3.15 & NM_008599 & Cxcl9 & 2.34 \\
NM_138648 & Olr1 & 3.10 & NM_026994 & Cryzll & 2.33 \\
NM_024198 & Gpx7 & 3.03 & NM_178628 & Spg3a & 2.33 \\
NM_007642 & Cd28 & 2.89 & NM_011331 & Ccll2 & 2.31 \\
NM_011364 & Sh2d1a & 2.80 & NM_008360 & Ill1 & 2.28 \\
NM_009141 & Cxcl5 & 2.76 & NM_009127 & Scdl & 2.20 \\
NM_011260 & Reg3g & 2.71 & NM_007574 & Clqg & 2.20 \\
NM_009417 & Tpo & 2.70 & NM_010877 & Ncf2 & 2.19 \\
NM_010343 & Gpx5 & 2.69 & NM_015790 & Icosl & 2.18 \\
NM_007851 & Defcr5 & 2.65 & NM_019450 & Illf6 & 2.17 \\
NM_008518 & Ltb & 2.63 & NM_009912 & Ccr1 & 2.17 \\
NM_023517 & Thfsf13 & 2.60 & NM_009140 & Cxcl2 & 2.15 \\
NM_126166 & Tlr3 & 2.59 & NM_025316 & Ndufb5 & 2.13
\end{tabular}


H. Kurita et al.

Table 7. (Continued).

\begin{tabular}{llcllc}
\hline Accession Number & Gene Name & Fold of change & Accession Number & Gene Name & Fold of change \\
\hline NM_010739 & Ly64 & 2.58 & NM_008622 & Mpv17 & 2.11 \\
NM_011347 & Selp & 2.57 & NM_009114 & S100a9 & 2.11 \\
NM_008320 & Icsbp1 & 2.54 & NM_010819 & Clecsf8 & 2.10 \\
NM_025523 & Ndufc1 & 2.53 & NM_018763 & Chst2 & 2.10 \\
NM_019977 & Aldrl6 & 2.53 & NM_019418 & Tnfsfl4 & 2.10 \\
NM_019728 & Defb4 & 2.52 & NM_013723 & Podxl & 2.09 \\
NM_019944 & Hlxb9 & 2.51 & NM_027320 & Ifi35 & 2.08 \\
NM_007762 & Crhrl & 2.49 & NM_009777 & Clqb & 2.07 \\
NM_008337 & Ifng & 2.49 & NM_009311 & Tac1 & 2.07 \\
NM_008699 & Nkx2-3 & 2.48 & NM_007946 & Epx & 2.06 \\
AK006243 & Ndufa3 & 2.47 & NM_021364 & Clecsf5 & 2.06 \\
NM_010072 & Dpm1 & 2.46 & NM_019577 & Ccl24 & 2.05 \\
NM_009854 & Cd7 & 2.43 & NM_011604 & Tlr6 & 2.04 \\
NM_008361 & Illb & 2.43 & NM_008501 & Lif & 2.04 \\
NM_007925 & Eln & 2.42 & NM_028717 & Als2 & 2.03 \\
NM_019932 & Cxcl4 & 2.42 & NM_008823 & Pfc & 2.03 \\
NM_054096 & Tirap & 2.41 & NM_021319 & Pglyrpl & 2.02 \\
NM_019507 & Tbx21 & 2.41 & NM_010369 & Gypa & 2.01 \\
NM_001190325 & Igl-5 & NM_021396 & Pdcd1lg2 & 2.01 \\
NM_021283 & Il4 & 2.37 & & &
\end{tabular}

Table 8. Down-regulated genes related to cellular defense in the mouse fetal liver following gestational Cd exposure.

\begin{tabular}{llcllc}
\hline Accession Number & Gene Name & Fold of change & Accession Number & Gene Name & Fold of change \\
\hline NM_010381 & $H 2-E a$ & 0.275 & NM_010382 & $H 2-E b 1$ & 0.496 \\
NM_011691 & Vav1 & 0.389 & NM_007806 & Cyba & 0.498 \\
NM_009857 & Cd8a & 0.442 & & & \\
\hline
\end{tabular}

Table 9. Up-regulated genes related to metabolism in the mouse fetal liver following gestational Cd exposure.

\begin{tabular}{llcllc}
\hline Accession Number & Gene Name & Fold of change & Accession Number & Gene Name & Fold of change \\
\hline NM_008537 & Amacr & 3.70 & NM_031159 & Apobecl & 2.42 \\
NM_053070 & Car7 & 3.45 & NM_008876 & Pld2 & 2.42 \\
NM_031202 & Tyrp1 & 3.40 & NM_021509 & Moxd1 & 2.38 \\
NM_027979 & Chit1 & 3.13 & NM_021460 & Lip1 & 2.38 \\
NM_001305982 & Coasy & 3.09 & NM_025881 & Luc7l & 2.36 \\
NM_011128 & Pnliprp2 & 3.09 & NM_011528 & Taldo1 & 2.36 \\
NM_026438 & Pyp & 3.09 & NM_008875 & Pld1 & 2.34 \\
NM_008062 & G6pdx & 3.07 & NM_023624 & Lrat & 2.34 \\
NM_011704 & Vnn1 & 3.04 & NM_011224 & Pygm & 2.34 \\
NM_009802 & Car6 & 3.02 & NM_145953 & Cth & 2.31 \\
NM_010338 & Gpr37 & 2.98 & NM_030017 & Rdh12 & 2.31 \\
NM_008078 & Gad2 & 2.98 & NM_013792 & Naglu & 2.30 \\
NM_022014 & Fn3k & 2.95 & NM_008325 & Idua & 2.30 \\
NM_025396 & Pgls & 2.84 & NM_016722 & Galns & 2.29 \\
NM_133653 & Mat1a & 2.79 & NM_008292 & Hsd17b4 & 2.29 \\
NM_019811 & Acas2 & 2.76 & NM_008185 & Grp10 & 2.29 \\
NM_022305 & B4galt1 & 2.76 & & & 2.29
\end{tabular}


Gene expression changes in fetal liver of gestational Cd-exposed mice

Table 9. (Continued).

\begin{tabular}{|c|c|c|c|c|c|}
\hline Accession Number & Gene Name & Fold of change & Accession Number & Gene Name & Fold of change \\
\hline NM_001172055 & Dhrs6 & 2.75 & NM_007433 & Akp5 & 2.28 \\
\hline NM_026408 & Sncaip & 2.72 & NM_008866 & Lypla1 & 2.26 \\
\hline NM_009949 & Cpt2 & 2.71 & NM_145569 & Mat $2 a$ & 2.26 \\
\hline NM_019477 & Acsl4 & 2.69 & NM_026644 & Agpat4 & 2.25 \\
\hline NM_019676 & Plcd & 2.66 & NM_031201 & Tsta3 & 2.25 \\
\hline NM_013463 & Gla & 2.65 & NM_013761 & Srr & 2.24 \\
\hline NM_134075 & Gmpr2 & 2.64 & NM_018757 & Nme6 & 2.24 \\
\hline NM_024188 & Oxct & 2.63 & NM_009230 & Soat1 & 2.24 \\
\hline AF178429 & AV071179 & 2.62 & NM_023175 & D16Ertd502e & 2.23 \\
\hline NM_013556 & Hprt & 2.62 & NM_009171 & Shmt1 & 2.20 \\
\hline NM_133900 & Psph & 2.62 & NM_028072 & Sulf2 & 2.19 \\
\hline NM_010952 & $\mathrm{Oaz} 2$ & 2.59 & NM_016772 & Ech1 & 2.19 \\
\hline NM_177823 & Ubash $3 a$ & 2.59 & NM_008291 & $H s d 17 b 3$ & 2.18 \\
\hline NM_011961 & Plod2 & 2.58 & NM_023697 & Rdh14 & 2.16 \\
\hline NM_009409 & Top $2 b$ & 2.57 & NM_016672 & $D d c$ & 2.16 \\
\hline NM_017465 & Sult $2 b 1$ & 2.57 & NM_013784 & Pign & 2.16 \\
\hline NM_018870 & Pgam2 & 2.56 & NM_033320 & Glce & 2.15 \\
\hline NM_025337 & Akr7a5 & 2.55 & NM_022033 & Oxct $2 a$ & 2.15 \\
\hline NM_013502 & Ctbpl & 2.54 & NM_028803 & Gbel & 2.15 \\
\hline NM_053184 & Ugt2al & 2.53 & NM_010362 & Gstol & 2.12 \\
\hline NM_020271 & $P d x p$ & 2.52 & NM_015804 & Atp11a & 2.11 \\
\hline NM_019957 & Dnase $2 b$ & 2.52 & NM_008079 & Galc & 2.11 \\
\hline NM_138595 & Gldc & 2.49 & NM_025295 & $B t d$ & 2.08 \\
\hline NM_173047 & Cbr3 & 2.49 & NM_027406 & Fthfd & 2.06 \\
\hline NM_019878 & Sult $1 b 1$ & 2.49 & NM_010422 & Hexb & 2.06 \\
\hline NM_025522 & Dhrs 7 & 2.45 & NM_008077 & Gadl & 2.04 \\
\hline NM_133198 & Pygl & 2.44 & NM_011514 & Suv39h1 & 2.03 \\
\hline NM_025922 & Itpa & 2.44 & NM_009286 & Sth2 & 2.03 \\
\hline NM_013624 & Otog & 2.43 & NM_027924 & $P d g f d$ & 2.03 \\
\hline
\end{tabular}

Table 10. Down-regulated genes related to metabolism in the mouse fetal liver following gestational Cd exposure.

\begin{tabular}{llcllc}
\hline Accession Number & Gene Name & Fold of change & Accession Number & Gene Name & Fold of change \\
\hline NM_134079 & Adk & 0.226 & NM_013847 & Gcat & 0.434 \\
NM_016807 & Sdcbp & 0.309 & NM_010893 & Neul & 0.462 \\
NM_178389 & Gale & 0.343 & NM_024243 & Fuca & 0.462 \\
NM_134188 & Mte1 & 0.401 & NM_008745 & Ntrk2 & 0.464 \\
NM_007512 & Atpi & 0.423 & NM_010233 & Fn1 & 0.481 \\
NM_134246 & Pte2a & 0.429 & NM_008000 & Fert2 & 0.487 \\
\hline
\end{tabular}


H. Kurita et al.

Table 11. Up-regulated genes related to transport in the mouse fetal liver following gestational Cd exposure.

\begin{tabular}{|c|c|c|c|c|c|}
\hline Accession Number & Gene Name & Fold of change & Accession Number & Gene Name & Fold of change \\
\hline NM_011743 & Zfp106 & 3.47 & NM_008999 & Rab23 & 2.39 \\
\hline NM_011388 & Slc10a2 & 3.41 & NM_016792 & Txnl & 2.38 \\
\hline NM_032397 & Kcnn1 & 3.30 & NM_019430 & Cacng3 & 2.38 \\
\hline NM_021520 & Slc28a2 & 3.25 & NM_011539 & Tbxas 1 & 2.38 \\
\hline NM_021291 & Slc7a9 & 3.20 & NM_013716 & $G 3 b p$ & 2.38 \\
\hline NM_025535 & Sara2 & 3.19 & NM_023537 & $R a b 3 b$ & 2.37 \\
\hline NM_029638 & $A b p 1$ & 3.16 & NM_007873 & $D o c 2 b$ & 2.37 \\
\hline NM_023348 & Snap29 & 3.14 & NM_008069 & Gabrbl & 2.36 \\
\hline NM_139229 & $\operatorname{Cog} 8$ & 3.11 & NM_016858 & $R a b 33 b$ & 2.36 \\
\hline NM_029394 & $\operatorname{Sn} \times 24$ & 3.11 & NM_011795 & Clqrf & 2.35 \\
\hline NM_024412 & Clcnkl & 3.11 & NM_010276 & Gem & 2.35 \\
\hline NM_019659 & Kcnj1 & 3.09 & NM_010472 & $H r b$ & 2.35 \\
\hline NM_033444 & Clic1 & 3.07 & NM_008948 & Psmc3 & 2.34 \\
\hline NM_153155 & $C l q l$ & 3.06 & NM_021492 & $A p 3 b 2$ & 2.34 \\
\hline NM_020574 & Kcne3 & 3.05 & NM_008097 & $G c d h$ & 2.33 \\
\hline NM_178703 & Slc6a1 & 3.04 & NM_010760 & Magoh & 2.33 \\
\hline NM_019792 & Cyp $3 a 25$ & 3.03 & NM_011598 & Fabp 9 & 2.32 \\
\hline NM_028011 & Srcasm & 3.01 & NM_024414 & Stx $1 b 2$ & 2.32 \\
\hline NM_008467 & Kpna4 & 3.00 & NM_021329 & Rangnrf & 2.32 \\
\hline NM_025505 & $B l z f 1$ & 2.97 & NM_176844 & Chrna5 & 2.32 \\
\hline NM_011878 & Tiam2 & 2.97 & NM_013569 & Kcnh2 & 2.31 \\
\hline NM_024448 & Rab12 & 2.96 & NM_008244 & $\mathrm{HgS}$ & 2.31 \\
\hline $\mathrm{AB} 006034$ & Cyp27b1 & 2.94 & NM_011075 & $A b c b 1 b$ & 2.30 \\
\hline NM_009996 & Cyp24al & 2.94 & NM_015751 & Abcel & 2.30 \\
\hline NM_019931 & Kcnd3 & 2.93 & NM_133950 & Kdelr1 & 2.30 \\
\hline NM_001163011 & Mup1 & 2.92 & NM_011254 & Rbpl & 2.30 \\
\hline NM_007899 & Ecm 1 & 2.92 & NM_021536 & Arht1 & 2.30 \\
\hline NM_010349 & Grik2 & 2.91 & NM_145559 & Slc2a9 & 2.29 \\
\hline NM_026887 & Apls 2 & 2.89 & NM_009579 & Slc30al & 2.29 \\
\hline NM_025807 & Slcl6a9 & 2.89 & NM_013885 & Clic4 & 2.29 \\
\hline NM_010595 & Kcnal & 2.87 & NM_027881 & Osbpl3 & 2.29 \\
\hline NM_027915 & $A p 2 b 1$ & 2.87 & NM_023732 & $A b c b 6$ & 2.28 \\
\hline NM_008642 & Mttp & 2.87 & NM_009944 & Cox7al & 2.28 \\
\hline NM_007503 & Fxyd2 & 2.85 & NM_011591 & Timm17b & 2.28 \\
\hline NM_011325 & Scnn $1 b$ & 2.85 & NM_007824 & Cyp7al & 2.27 \\
\hline NM_009198 & Slc17a1 & 2.84 & NM_144593 & Rhebll & 2.27 \\
\hline NM_133199 & $\operatorname{Scn} 4 a$ & 2.84 & NM_029158 & Cnbp2 & 2.26 \\
\hline NM_011773 & Slc30a3 & 2.83 & NM_031251 & Ctns & 2.26 \\
\hline NM_011256 & Pitpnm2 & 2.80 & NM_009728 & Atp10a & 2.26 \\
\hline NM_011396 & Slc $22 a 5$ & 2.79 & NM_029019 & Stard6 & 2.25 \\
\hline NM_026532 & Nutf2 & 2.79 & NM_023134 & Sftpa & 2.25 \\
\hline NM_011322 & Scn $1 b$ & 2.78 & NM_016801 & Stxla & 2.25 \\
\hline NM_010298 & Glrb & 2.78 & NM_009604 & Chrng & 2.25 \\
\hline NM_016703 & Preb & 2.77 & NM_011076 & Abcbla & 2.25 \\
\hline NM_009603 & Chrne & 2.76 & NM_031261 & Fthl17 & 2.24 \\
\hline NM_019432 & $\operatorname{Prl}$ & 2.76 & NM_020599 & Rlbp1 & 2.24 \\
\hline NM_019722 & Arl2 & 2.76 & NM_025381 & Atp6v1f & 2.24 \\
\hline NM_022813 & Scamp2 & 2.76 & NM_001081056 & Xpot & 2.24 \\
\hline NM_026678 & Blvra & 2.76 & NM_022317 & Slc28a3 & 2.23 \\
\hline
\end{tabular}


Gene expression changes in fetal liver of gestational Cd-exposed mice

Table 11. (Continued).

\begin{tabular}{|c|c|c|c|c|c|}
\hline Accession Number & Gene Name & Fold of change & Accession Number & Gene Name & Fold of change \\
\hline NM_021789 & Trappc4 & 2.75 & NM_031194 & Slc22a8 & 2.22 \\
\hline NM_133225 & $A c b d 3$ & 2.75 & NM_019761 & Nxt1 & 2.22 \\
\hline NM_019692 & Rit2 & 2.75 & NM_008430 & Kcnkl & 2.22 \\
\hline NM_009004 & Kif20a & 2.75 & NM_009466 & $U g d h$ & 2.21 \\
\hline NM_021375 & $R h b g$ & 2.74 & NM_029466 & Arls & 2.21 \\
\hline NM_027889 & Vps11 & 2.74 & NM_008070 & Gabrb2 & 2.21 \\
\hline NM_025914 & Actr6 & 2.74 & NM_027398 & Kcnip1 & 2.21 \\
\hline NM_009320 & Slc6a6 & 2.73 & NM_007515 & Slc7a3 & 2.20 \\
\hline NM_024439 & $H 47$ & 2.73 & NM_019566 & Arhg & 2.20 \\
\hline NM_026180 & $A b c g 8$ & 2.73 & NM_026677 & Rab13 & 2.20 \\
\hline NM_026130 & Srpr & 2.73 & NM_028388 & Ndufve & 2.20 \\
\hline NM_010586 & Itpr 5 & 2.72 & NM_007389 & Chrnal & 2.20 \\
\hline NM_007819 & Сур3а13 & 2.71 & NM_024287 & Rab6 & 2.20 \\
\hline NM_019741 & Slc $2 a 5$ & 2.71 & NM_007760 & Crat & 2.20 \\
\hline NM_020270 & Scamp 5 & 2.70 & NM_016857 & Exoc7 & 2.19 \\
\hline NM_021530 & Slc $4 a 8$ & 2.69 & NM_008227 & Hcn3 & 2.19 \\
\hline NM_008428 & Kcnj8 & 2.68 & NM_001081048 & Slc25a18 & 2.19 \\
\hline NM_021471 & Slcolcl & 2.67 & NM_007582 & Cacngl & 2.18 \\
\hline NM_028052 & Synpr & 2.67 & NM_028711 & Slc25a27 & 2.18 \\
\hline NM_019923 & Itpr 2 & 2.66 & NM_021879 & $p$ & 2.18 \\
\hline NM_009405 & Tnni2 & 2.65 & NM_023671 & Clnsla & 2.17 \\
\hline NM_031195 & Msrl & 2.65 & NM_011485 & Star & 2.17 \\
\hline NM_026298 & Wim & 2.65 & NM_011027 & $P 2 r x 7$ & 2.17 \\
\hline NM_001081049 & Mll & 2.63 & NM_028064 & Slc39a4 & 2.17 \\
\hline NM_010600 & Kcnh1 & 2.63 & NM_001160139 & Kcnq5 & 2.16 \\
\hline NM_030556 & Slc19a3 & 2.63 & NM_026200 & Kcnv1 & 2.16 \\
\hline NM_009203 & Slc22a12 & 2.63 & NM_027439 & Atp6ap2 & 2.15 \\
\hline NM_008165 & Grial & 2.62 & NM_021710 & Ap4s1 & 2.15 \\
\hline NM_011405 & Slc $7 a 7$ & 2.61 & NM_008998 & Rab17 & 2.15 \\
\hline NM_013900 & Mfi2 & 2.59 & NM_008172 & Grin2d & 2.14 \\
\hline NM_008031 & Fmrl & 2.59 & NM_019510 & $\operatorname{Trpc} 3$ & 2.14 \\
\hline NM_026468 & Atp $5 g 2$ & 2.59 & NM_007618 & Serpina6 & 2.14 \\
\hline NM_019720 & $A W 049681$ & 2.59 & NM_016755 & Atp5j & 2.13 \\
\hline NM_025826 & Acadsb & 2.58 & NM_009306 & Syt 1 & 2.13 \\
\hline NM_030888 & C1qtnf3 & 2.57 & NM_009928 & Coll5al & 2.13 \\
\hline NM_008076 & Gabrr2 & 2.56 & NM_030683 & Slc14a2 & 2.13 \\
\hline NM_001081084 & Cubn & 2.55 & NM_021415 & Cacnalh & 2.13 \\
\hline NM_013415 & Atp1b2 & 2.54 & NM_009005 & Rab7 & 2.13 \\
\hline NM_010354 & Gsn & 2.54 & NM_007382 & Acadm & 2.12 \\
\hline NM_031173 & Cacnbl & 2.53 & NM_009720 & Atox 1 & 2.12 \\
\hline NM_008072 & Gabrd & 2.53 & NM_013454 & Abcal & 2.11 \\
\hline NM_011504 & Stxbp3 & 2.53 & NM_008713 & Nos 3 & 2.11 \\
\hline NM_029673 & Immt & 2.52 & NM_029505 & Ap3m2 & 2.11 \\
\hline NM_008423 & Kcnd1 & 2.52 & NM_016752 & Slc35b1 & 2.11 \\
\hline NM_008600 & Mip & 2.51 & NM_007885 & Slc26a2 & 2.11 \\
\hline NM_028942 & Slco6c1 & 2.51 & NM_007807 & $C y b b$ & 2.10 \\
\hline NM_009994 & Cyplb1 & 2.50 & NM_027862 & Atp $5 h$ & 2.10 \\
\hline NM_019701 & Clcnkb & 2.50 & NM_019696 & Cpxm1 & 2.08 \\
\hline NM_018804 & Syt11 & 2.50 & NM_010215 & $I l 4 i 1$ & 2.08 \\
\hline
\end{tabular}


H. Kurita et al.

Table 11. (Continued).

\begin{tabular}{|c|c|c|c|c|c|}
\hline Accession Number & Gene Name & Fold of change & Accession Number & Gene Name & Fold of change \\
\hline NM_013790 & $A b c c 5$ & 2.50 & NM_053204 & Rab6ip2 & 2.08 \\
\hline NM_023214 & Slc30a7 & 2.49 & NM_008596 & $\operatorname{Mg} 29$ & 2.07 \\
\hline NM_029655 & $\operatorname{Sn} x 7$ & 2.49 & NM_009941 & Cox4i1 & 2.07 \\
\hline NM_008418 & Kcna3 & 2.48 & NM_022992 & Arl6ip5 & 2.07 \\
\hline NM_009659 & Alox $12 b$ & 2.48 & NM_138660 & Casc3 & 2.07 \\
\hline NM_024458 & $P d c$ & 2.48 & NM_009395 & Tnfaipl & 2.07 \\
\hline NM_008997 & $R a b 11 b$ & 2.48 & NM_011389 & Slcl2al & 2.06 \\
\hline NM_019758 & Mtch2 & 2.47 & NM_007457 & Apls1 & 2.05 \\
\hline NM_030239 & Abcg3 & 2.47 & NM_010250 & Gabral & 2.05 \\
\hline NM_029600 & $A b c c 3$ & 2.47 & NM_013635 & Sypl & 2.05 \\
\hline NM_007810 & Cyp19a1 & 2.46 & NM_021284 & Kras2 & 2.05 \\
\hline NM_021370 & Accn 5 & 2.46 & NM_126165 & $V p s 4 a$ & 2.04 \\
\hline NM_027008 & Kctd 5 & 2.45 & NM_019415 & Slc12a3 & 2.04 \\
\hline NM_011310 & S100a3 & 2.45 & NM_026610 & $N d u f b 4$ & 2.04 \\
\hline NM_007480 & $\operatorname{Arf} 5$ & 2.45 & NM_026998 & Snx6 & 2.03 \\
\hline NM_008851 & Pitpnm & 2.44 & NM_013895 & Timm 9 & 2.03 \\
\hline NM_023126 & $R a b 8 a$ & 2.44 & NM_152220 & Stx 3 & 2.03 \\
\hline NM_021544 & $\operatorname{Scn} 5 a$ & 2.43 & NM_007825 & Cyp $7 b 1$ & 2.03 \\
\hline NM_021547 & Stard3 & 2.43 & NM_133969 & Cyp $4 v 3$ & 2.03 \\
\hline NM_028238 & Rab38 & 2.42 & NM_028712 & $\operatorname{Rap} 2 b$ & 2.01 \\
\hline NM_018768 & Stx 8 & 2.41 & NM_009134 & Scn10a & 2.01 \\
\hline NM_023743 & Eif4enif1 & 2.40 & NM_027352 & Gorasp2 & 2.01 \\
\hline NM_009942 & Cox $5 b$ & 2.39 & NM_025573 & Sfrs 9 & 2.00 \\
\hline NM_019502 & Fxcl & 2.39 & NM_010599 & Kcnab3 & 2.00 \\
\hline
\end{tabular}

Table 12. Down-regulated genes related to transport in the mouse fetal liver following gestational Cd exposure.

\begin{tabular}{llcllc}
\hline Accession Number & Gene Name & Fold of change & Accession Number & Gene Name & Fold of change \\
\hline NM_080467 & Atp6v0a4 & 0.331 & NM_009931 & Col4al & 0.452 \\
NM_009930 & Col3a1 & 0.349 & NM_011403 & Slc4a1 & 0.453 \\
NM_025823 & Pcyox1 & 0.384 & NM_007823 & Cyp4b1 & 0.455 \\
NM_001081097 & Grik3 & 0.393 & NM_133189 & Cacng7 & 0.486 \\
NM_007854 & Slc29a2 & 0.395 & NM_133254 & Slc5a2 & 0.486 \\
NM_022327 & Ralb & 0.408 & NM_138955 & Abcg4 & 0.489 \\
NM_018762 & Gp9 & 0.428 & NM_011436 & Sorl1 & 0.492 \\
NM_148944 & Chrnb4 & 0.431 & NM_007506 & Atp5g1 & 0.494 \\
NM_007742 & Colla1 & 0.433 & NM_013683 & Tap1 & 0.495 \\
NM_028247 & Slc16a10 & 0.444 & NM_019552 & Abcb10 & 0.499 \\
NM_026553 & Yif1 & 0.444 & & &
\end{tabular}


Gene expression changes in fetal liver of gestational Cd-exposed mice

Table 13. Up-regulated genes related to transcription in the mouse fetal liver following gestational Cd exposure.

\begin{tabular}{|c|c|c|c|c|c|}
\hline Accession Number & Gene Name & Fold of change & Accession Number & Gene Name & Fold of change \\
\hline NM_010789 & Meis1 & 3.37 & NM_029999 & $L b h$ & 2.39 \\
\hline NM_007498 & Atf3 & 3.34 & NM_023672 & Ssbp3 & 2.39 \\
\hline NM_007924 & Ell & 3.30 & NM_011603 & Tbpl1 & 2.38 \\
\hline NM_053254 & Tle6 & 3.29 & NM_013744 & Zfp $354 b$ & 2.38 \\
\hline NM_007959 & Etsrp 71 & 3.23 & NM_027139 & Taf9 & 2.38 \\
\hline NM_007471 & $A p p$ & 3.15 & NM_008499 & Lhx5 & 2.37 \\
\hline NM_007855 & Twist2 & 3.10 & NM_025307 & $N r b f 2$ & 2.37 \\
\hline NM_027326 & Mllt3 & 3.02 & NM_011294 & Rpo2tcl & 2.36 \\
\hline NM_013926 & $C b x 8$ & 2.96 & NM_010467 & Hoxd1 & 2.35 \\
\hline NM_053170 & Trim33 & 2.95 & NM_023729 & Gasz & 2.34 \\
\hline NM_013476 & $A r$ & 2.92 & NM_145632 & Polr $2 h$ & 2.34 \\
\hline NM_009234 & Sox 11 & 2.89 & NM_009322 & Tbrl & 2.34 \\
\hline NM_008692 & Nfyc & 2.89 & NM_007880 & Arid $3 a$ & 2.33 \\
\hline NM_145831 & Dmrt2 & 2.89 & NM_130893 & Scrt1 & 2.33 \\
\hline NM_018809 & Ptfla & 2.88 & NM_010452 & Hoxa3 & 2.33 \\
\hline NM_010894 & Neurod1 & 2.88 & NM_019660 & Mycbp & 2.32 \\
\hline NM_010136 & Eomes & 2.86 & NM_020005 & Pcaf & 2.32 \\
\hline NM_011551 & Ubtf & 2.85 & NM_053213 & Mad3 & 2.32 \\
\hline NM_020584 & Terf2ip & 2.83 & NM_028245 & Zfpl31 & 2.31 \\
\hline NM_011037 & $\operatorname{Pax} 2$ & 2.83 & NM_009116 & Prrx2 & 2.31 \\
\hline NM_011541 & Tceal & 2.81 & NM_010919 & $N k x 2-2$ & 2.31 \\
\hline NM_021318 & Fhl5 & 2.80 & NM_010574 & $\operatorname{Irx} 2$ & 2.30 \\
\hline NM_019460 & Sfmbt1 & 2.79 & NM_019994 & Garnll & 2.30 \\
\hline NM_016793 & Zfp 98 & 2.76 & NM_013519 & Foxc2 & 2.30 \\
\hline NM_009578 & Zfpnlal & 2.76 & NM_133971 & Ankrd10 & 2.30 \\
\hline NM_013827 & $M t f 2$ & 2.75 & NM_010426 & Foxfla & 2.29 \\
\hline NM_011658 & Twist1 & 2.75 & NM_026496 & $T c f c p 2 l 3$ & 2.28 \\
\hline NM_011499 & Strap & 2.75 & NM_028041 & $D d x 54$ & 2.28 \\
\hline NM_027949 & $P h f 7$ & 2.75 & NM_011307 & Rxrip110 & 2.27 \\
\hline NM_020483 & Hcngp & 2.75 & NM_008652 & Mybl2 & 2.27 \\
\hline NM_010692 & $L b \times 2 h$ & 2.74 & NM_008566 & Mcm5 & 2.26 \\
\hline NM_020558 & Cld & 2.74 & NM_010938 & Nrfl & 2.26 \\
\hline NM_013874 & Neud4 & 2.74 & NM_025891 & Smarcd3 & 2.24 \\
\hline NM_011584 & $\operatorname{Nr} 1 d 2$ & 2.73 & NM_007615 & Catns & 2.24 \\
\hline NM_175303 & Sall4 & 2.73 & NM_009547 & Zfpl61 & 2.23 \\
\hline NM_008735 & Nripl & 2.71 & NM_016861 & Pdlim 1 & 2.23 \\
\hline NM_020255 & Scandl & 2.70 & NM_008393 & $\operatorname{Irx} 3$ & 2.21 \\
\hline NM_010158 & Khdrbs 3 & 2.70 & NM_054039 & Foxp3 & 2.20 \\
\hline NM_130869 & $\operatorname{Og} 2 x$ & 2.68 & NM_008543 & Madh7 & 2.20 \\
\hline NM_013665 & Shox 2 & 2.68 & NM_008271 & Hoxc 5 & 2.19 \\
\hline NM_028709 & Btbd11 & 2.66 & NM_013720 & $M g a$ & 2.19 \\
\hline NM_013915 & $Z f p 238$ & 2.65 & NM_174852 & Phf12 & 2.18 \\
\hline NM_010151 & $N r 2 f 1$ & 2.65 & NM_009360 & Tfam & 2.18 \\
\hline NM_178280 & Sall3 & 2.65 & NM_011550 & Tcfl4 & 2.18 \\
\hline NM_010592 & Jund1 & 2.65 & NM_181650 & $\operatorname{Prdm} 4$ & 2.17 \\
\hline NM_008274 & Hoxd12 & 2.64 & NM_026107 & Zfp535 & 2.17 \\
\hline NM_010420 & Hesx 1 & 2.64 & NM_008506 & Lmycl & 2.17 \\
\hline NM_008900 & Pou $3 f 3$ & 2.64 & NM_010118 & Egr2 & 2.17 \\
\hline NM_010909 & Nfkbill & 2.61 & NM_011813 & Fiz1 & 2.17 \\
\hline
\end{tabular}


H. Kurita et al.

Table 13. (Continued).

\begin{tabular}{|c|c|c|c|c|c|}
\hline Accession Number & Gene Name & Fold of change & Accession Number & Gene Name & Fold of change \\
\hline NM_008684 & $\mathrm{Neol}$ & 2.59 & NM_023814 & Tbx18 & 2.16 \\
\hline NM_016967 & Olig2 & 2.58 & NM_027485 & Crsp7 & 2.16 \\
\hline NM_008950 & Psmc5 & 2.58 & NM_011605 & Tmpo & 2.16 \\
\hline NM_011136 & Pou2af1 & 2.56 & NM_015758 & Foxe3 & 2.15 \\
\hline NM_010895 & Neurod2 & 2.56 & NM_008899 & Pou $3 f 2$ & 2.15 \\
\hline NM_027547 & $\operatorname{Prdm} 5$ & 2.56 & NM_008657 & Myf6 & 2.14 \\
\hline NM_011980 & Zfp 146 & 2.55 & NM_008716 & Notch 3 & 2.13 \\
\hline NM_207671 & Zfp 318 & 2.55 & NM_025444 & Taf13 & 2.13 \\
\hline NM_009541 & Zfp 100 & 2.54 & NM_008849 & Pit1 & 2.12 \\
\hline NM_009545 & Rnf110 & 2.53 & NM_023755 & $T c f c p 2 l 1$ & 2.11 \\
\hline NM_010832 & Msl31 & 2.52 & NM_008260 & Foxa3 & 2.11 \\
\hline NM_017373 & Nfil3 & 2.50 & NM_008262 & Onecut1 & 2.10 \\
\hline NM_013684 & $T b p$ & 2.50 & NM_027347 & Crsp3 & 2.10 \\
\hline NM_017375 & Ostf1 & 2.50 & NM_023472 & Ankra2 & 2.08 \\
\hline NM_009122 & Satbl & 2.49 & NM_010449 & Hoxal & 2.08 \\
\hline NM_011600 & Tle4 & 2.49 & NM_011850 & $\mathrm{NrOb2}$ & 2.08 \\
\hline NM_021901 & $T l x 1$ & 2.48 & NM_009123 & $N k x 1-2$ & 2.07 \\
\hline NM_008814 & Ipfl & 2.48 & NM_009108 & $N r 1 h 4$ & 2.07 \\
\hline NM_024124 & Hdac9 & 2.47 & NM_009550 & $Z f p 2$ & 2.06 \\
\hline NM_001042499 & Rabl3 & 2.46 & NM_028868 & Cxxcl & 2.05 \\
\hline NM_026374 & Ilf2 & 2.46 & NM_022009 & Fliih & 2.05 \\
\hline NM_01011021 & $O t p$ & 2.45 & NM_178392 & Snapcl & 2.05 \\
\hline NM_011565 & Tead2 & 2.44 & NM_030690 & Rail4 & 2.05 \\
\hline NM_026383 & Pnrc2 & 2.44 & NM_011935 & Esrrg & 2.05 \\
\hline NM_019791 & Magedl & 2.44 & NM_033652 & Lmxla & 2.04 \\
\hline NM_011066 & Per2 & 2.44 & NM_010836 & $M s \times 3$ & 2.04 \\
\hline NM_009329 & Zfp $354 a$ & 2.43 & NM_010057 & Dlx6 & 2.03 \\
\hline NM_010445 & $H m x 1$ & 2.42 & NM_008700 & $N k \times 2-5$ & 2.03 \\
\hline NM_021462 & Mknk2 & 2.42 & NM_183248 & $N k x 6-2$ & 2.03 \\
\hline NM_023907 & Foxil & 2.42 & NM_028932 & Eaf1 & 2.03 \\
\hline NM_027689 & $R f x 4$ & 2.41 & NM_019446 & Barhll & 2.02 \\
\hline NM_013672 & Spl & 2.41 & NM_011135 & Cnot7 & 2.02 \\
\hline
\end{tabular}

Table 14. Down-regulated genes related to transcription in the mouse fetal liver following gestational Cd exposure.

\begin{tabular}{llcllc}
\hline Accession Number & Gene Name & Fold of change & Accession Number & Gene Name & Fold of change \\
\hline NM_001114140 & Tcf20 & 0.160 & NM_011605 & Tmpo & 0.399 \\
NM_010446 & Foxa2 & 0.232 & NM_011547 & Tcfap2a & 0.410 \\
NM_201644 & Ugt1a12 & 0.325 & NM_008252 & Hmgb2 & 0.435 \\
NM_013601 & Msx2 & 0.343 & NM_183298 & Foxe1 & 0.452 \\
NM_008265 & Hoxa4 & 0.369 & NM_009554 & Zfp37 & 0.452 \\
NM_010127 & Pou6f1 & 0.378 & NM_007531 & Bcap37 & 0.457 \\
NM_010226 & Fkhl18 & 0.381 & NM_010212 & Fhl2 & 0.458 \\
NM_010365 & Gtf2i & 0.387 & NM_011419 & Jarid1d & 0.495 \\
NM_011278 & Rnf4 & 0.389 & NM_011139 & Pou2f3 & 0.498 \\
NM_009343 & Phf1 & 0.392 & & &
\end{tabular}


Gene expression changes in fetal liver of gestational Cd-exposed mice

Table 15. Up-regulated genes related to signal transduction in the mouse fetal liver following gestational Cd exposure.

\begin{tabular}{|c|c|c|c|c|c|}
\hline Accession Number & Gene Name & Fold of change & Accession Number & Gene Name & Fold of change \\
\hline NM_011113 & Plaur & 3.40 & NM_009920 & Cnil & 2.45 \\
\hline NM_025609 & Map3k7ip1 & 3.34 & NM_008368 & $I l 2 r b$ & 2.45 \\
\hline NM_009314 & Tacr 2 & 3.33 & NM_146611 & MOR171-16 & 2.45 \\
\hline NM_007560 & Bmprlb & 3.23 & NM_008809 & Pdgfrb & 2.44 \\
\hline NM_015742 & Myo9b & 3.22 & NM_027763 & Treml1 & 2.44 \\
\hline NM_009706 & Arhgap5 & 3.16 & NM_031873 & Tas $1 r 2$ & 2.43 \\
\hline NM_025658 & $M s 4 a 4 d$ & 3.16 & NM_146798 & MOR163-1 & 2.43 \\
\hline NM_023121 & Gngt2 & 3.14 & NM_146745 & MOR171-12 & 2.42 \\
\hline NM_007641 & $M s 4 a 1$ & 3.13 & NM_007781 & Csf $2 r b 2$ & 2.42 \\
\hline NM_054040 & Tulp4 & 3.12 & NM_007407 & Adcyap1r1 & 2.41 \\
\hline NM_013530 & Gnb3 & 3.06 & NM_007726 & Cnrl & 2.41 \\
\hline NM_007721 & Gpr2 & 3.05 & NM_008315 & $H t r 7$ & 2.39 \\
\hline NM_013487 & $C d 3 d$ & 3.00 & NM_010320 & Gng8 & 2.39 \\
\hline NM_009063 & $\operatorname{Rgs} 5$ & 2.99 & NM_020265 & $D k k 2$ & 2.38 \\
\hline NM_010301 & Gnall & 2.98 & NM_012003 & Cops $7 a$ & 2.37 \\
\hline NM_013767 & Csnkle & 2.94 & NM_009290 & Wnt $8 a$ & 2.37 \\
\hline NM_011943 & Map $2 k 6$ & 2.94 & NM_020598 & Olfr 17 & 2.37 \\
\hline NM_013523 & Fshr & 2.92 & NM_008308 & Htrla & 2.37 \\
\hline NM_008058 & $F z d 8$ & 2.92 & NM_007420 & Adrb2 & 2.36 \\
\hline NM_019476 & Olfr159 & 2.92 & NM_206897 & Olfr6 & 2.34 \\
\hline NM_027677 & Gpr39 & 2.91 & NM_008043 & Frat1 & 2.33 \\
\hline NM_022012 & Map3k11 & 2.91 & NM_008141 & Gnat2 & 2.33 \\
\hline NM_019958 & $\operatorname{Rgs} 17$ & 2.90 & NM_015759 & $F g d 3$ & 2.32 \\
\hline NM_021368 & Ors16 & 2.90 & NM_007865 & Dll1 & 2.31 \\
\hline NM_007764 & Crkl & 2.90 & NM_011798 & Xcrl & 2.29 \\
\hline NM_025758 & Asb17 & 2.88 & NM_033269 & Chrm3 & 2.29 \\
\hline NM_027571 & P2ry 12 & 2.88 & NM_008965 & Ptger4 & 2.27 \\
\hline NM_028450 & Gulp1 & 2.86 & NM_007538 & Opn1sw & 2.27 \\
\hline NM_010200 & Fgfl3 & 2.84 & NM_013619 & Olfr67 & 2.25 \\
\hline NM_008174 & Grm8 & 2.84 & NM_010102 & $E d g 6$ & 2.25 \\
\hline NM_025285 & Stmn 2 & 2.82 & NM_008762 & Olfr 15 & 2.24 \\
\hline NM_133485 & Ppp1r14c & 2.82 & NM_009518 & Wnt10a & 2.23 \\
\hline NM_146746 & MOR171-11 & 2.82 & NM_021280 & Plcg1 & 2.23 \\
\hline NM_007863 & Mpp3 & 2.81 & NM_021381 & Gpr73 & 2.23 \\
\hline NM_022881 & $\operatorname{Rgs} 18$ & 2.77 & NM_147074 & Ors 19 & 2.23 \\
\hline NM_009759 & $B m x$ & 2.77 & NM_021457 & $F z d 1$ & 2.22 \\
\hline NM_010203 & $F g f 5$ & 2.75 & NM_009539 & Zap70 & 2.20 \\
\hline NM_019492 & $\operatorname{Rgs} 3$ & 2.75 & NM_145066 & Gpr85 & 2.20 \\
\hline NM_080557 & Snx4 & 2.74 & NM_011925 & $C d 97$ & 2.19 \\
\hline NM_019653 & Wsb1 & 2.72 & NM_009962 & Gpr44 & 2.19 \\
\hline NM_020501 & Tas2r105 & 2.71 & NM_029716 & Chnl & 2.18 \\
\hline NM_021892 & Rfrp & 2.71 & NM_021921 & Mapk8ip2 & 2.18 \\
\hline NM_010569 & Invs & 2.70 & NM_032399 & Gpr87 & 2.17 \\
\hline NM_021391 & Ppplr1a & 2.70 & NM_010254 & Galr2 & 2.17 \\
\hline NM_011682 & Utrn & 2.68 & NM_146614 & MOR171-13 & 2.14 \\
\hline NM_027965 & Gpr160 & 2.64 & NM_026446 & Rgs19 & 2.13 \\
\hline NM_019933 & Ptpn4 & 2.64 & NM_028736 & Grip1 & 2.13 \\
\hline NM_009939 & Cops 2 & 2.64 & NM_009168 & Shd & 2.13 \\
\hline NM_020510 & $F z d 2$ & 2.61 & NM_021609 & Ccbp2 & 2.11 \\
\hline
\end{tabular}


H. Kurita et al.

Table 15. (Continued).

\begin{tabular}{llllll}
\hline Accession Number & Gene Name & Fold of change & Accession Number & Gene Name & Fold of change \\
\hline NM_019917 & V2rlb & 2.60 & NM_007577 & C5rl & 2.10 \\
NM_020621 & P2ry4 & 2.60 & NM_011718 & Wnt10b & 2.09 \\
NM_007780 & Csf2rb1 & 2.59 & NM_011529 & Tank & 2.09 \\
NM_007427 & Agrp & 2.59 & NM_019404 & Avpr2 & 2.09 \\
NM_013867 & Bcar3 & 2.58 & NM_009519 & Wnt11 & 2.08 \\
NM_011756 & Zfp36 & 2.57 & NM_009848 & Entpd1 & 2.08 \\
NM_013521 & Fpr1 & 2.57 & NM_008356 & Il13ra2 & 2.08 \\
NM_008488 & Arhgef1 & 2.57 & NM_007720 & Ccr8 & 2.07 \\
NM_028724 & Rin2 & 2.55 & NM_010437 & Hivep2 & 2.05 \\
NM_018884 & Semcap3 & 2.55 & NM_146456 & MOR256-29 & 2.05 \\
NM_008140 & Gnat1 & 2.55 & NM_007718 & Ccrll1 & 2.04 \\
NM_019677 & Plcb1 & 2.52 & NM_008805 & Pde6a & 2.04 \\
NM_011924 & Avprlb & 2.52 & NM_008507 & Lnk & 2.03 \\
NM_030712 & Cxcr6 & 2.51 & NM_010130 & Emr1 & 2.03 \\
NM_011083 & Pik3c2a & 2.51 & NM_011268 & Rgs9 & 2.02 \\
NM_009835 & Ccr6 & 2.50 & NM_007395 & Acvrlb & 2.02 \\
NM_031380 & Fstl3 & 2.50 & NM_007878 & Drd4 & 2.02 \\
NM_013912 & Apln & 2.48 & NM_009827 & Cckar & 2.02 \\
NM_011941 & Mapkbp1 & 2.48 & NM_011104 & Prkce & 2.02 \\
NM_010341 & Gpr66 & 2.48 & NM_009914 & Ccr3 & 2.01 \\
NM_008747 & Ntsr2 & 2.47 & NM_001003685 & Ghrhr & 2.01 \\
NM_009216 & Sstr1 & 2.46 & NM_008154 & Gpr3 & 2.01 \\
NM_007974 & F2rl1 & 2.46 & Gpr12 & 2.01 \\
\hline
\end{tabular}

Table 16. Down-regulated genes related to signal transduction in the mouse fetal liver following gestational Cd exposure.

\begin{tabular}{|c|c|c|c|c|c|}
\hline Accession Number & Gene Name & Fold of change & Accession Number & Gene Name & Fold of change \\
\hline NM_146420 & MOR106-3 & 0.159 & NM_010342 & $G p r 7$ & 0.444 \\
\hline NM_146322 & MOR183-8 & 0.221 & NM_146777 & MOR110-2 & 0.445 \\
\hline NM_009630 & Adora $2 a$ & 0.350 & NM_146902 & MOR233-3 & 0.449 \\
\hline NM_146658 & $M O R 177-4$ & 0.351 & NM_207154 & MOR179-4 & 0.451 \\
\hline NM_080856 & Asb14 & 0.354 & NM_146827 & MOR162-1 & 0.455 \\
\hline NM_026270 & Akt1s 1 & 0.371 & NM_146904 & Olfr 72 & 0.456 \\
\hline NM_009487 & $V 2 r 11$ & 0.378 & NM_011634 & Traip & 0.456 \\
\hline NM_172285 & Plcg2 & 0.401 & NM_146989 & MOR127-1 & 0.462 \\
\hline NM_145848 & V1re13 & 0.412 & NM_008763 & Olfr 16 & 0.470 \\
\hline NM_146879 & MOR275-1 & 0.415 & NM_146270 & $M O R 267-16$ & 0.478 \\
\hline NM_146378 & MOR114-11 & 0.417 & NM_146840 & MOR42-1 & 0.481 \\
\hline NM_146402 & MOR245-7 & 0.423 & NM_147077 & MOR13-2 & 0.482 \\
\hline NM_146934 & Olfr 46 & 0.426 & NM_020510 & $F z d 2$ & 0.485 \\
\hline NM_146329 & MOR13-6 & 0.439 & NM_146576 & MOR120-1 & 0.489 \\
\hline NM_013523 & $F s h r$ & 0.442 & NM_010589 & Jak3 & 0.494 \\
\hline NM_207153 & MOR275-5 & 0.443 & NM_008312 & $\operatorname{Htr} 2 c$ & 0.496 \\
\hline
\end{tabular}


Gene expression changes in fetal liver of gestational Cd-exposed mice

Table 17. Up-regulated genes related to metal homeostasis in the mouse fetal liver following gestational Cd exposure.

\begin{tabular}{llcllc}
\hline Accession Number & Gene Name & Fold of change & Accession Number & Gene Name & Fold of change \\
\hline NM_028031 & Hip14l & 3.43 & NM_172121 & Zc3hdc3 & 2.48 \\
NM_133738 & Antxr2 & 2.76 & NM_023266 & Zfp120 & 2.47 \\
NM_019831 & Zfp261 & 2.72 & NM_019969 & Plag1 & 2.47 \\
NM_011746 & Mkrn3 & 2.71 & NM_025428 & Zdhhc12 & 2.46 \\
NM_028379 & Zdhhc4 & 2.71 & NM_020594 & Fliz1 & 2.45 \\
NM_008631 & Mt4 & 2.62 & NM_030684 & Trim34 & 2.43 \\
NM_133228 & Zfp72 & 2.60 & NM_009551 & Zfp216 & 2.43 \\
NM_024256 & B3Gat3 & 2.59 & NM_008114 & Gfilb & 2.37 \\
NM_028356 & Zfp50 & 2.59 & NM_212438 & Wiz & 2.37 \\
NM_011231 & Rabggtb & 2.56 & NM_030706 & Trim2 & 2.36 \\
NM_031172 & Trim17 & 2.56 & NM_053166 & Trim7 & 2.33 \\
NM_026796 & Smyd2 & 2.55 & NM_024231 & Zfpl1 & 2.31 \\
NM_013866 & Zfp385 & 2.52 & NM_018880 & Trim3 & 2.31 \\
NM_013602 & Mt1 & 2.51 & NM_020267 & Trim44 & 2.15 \\
NM_029104 & Zmynd17 & 2.50 & NM_026856 & D5Ertd689e & 2.13 \\
NM_207176 & Tes & 2.49 & NM_030219 & Trim42 & 2.13 \\
NM_011845 & Mid2 & 2.49 & NM_026021 & Zmynd19 & 2.12 \\
\hline
\end{tabular}

Table 18. Down-regulated genes related to metal homeostasis in the mouse fetal liver following gestational $\mathrm{Cd}$ exposure.

\begin{tabular}{llcllc}
\hline Accession Number & Gene Name & Fold of change & Accession Number & Gene Name & Fold of change \\
\hline NM_025920 & Thap4 & 0.286 & NM_021253 & Trim39 & 0.447 \\
NM_053100 & Trim8 & 0.412 & & &
\end{tabular}

Table 19. Up-regulated genes related to the ubiquitin-proteasome system in the mouse fetal liver following gestational Cd exposure.

\begin{tabular}{llcllc}
\hline Accession Number & Gene name & Fold of change & Accession Number & Gene name & Fold of change \\
\hline NM_011670 & Uchl1 & 3.20 & NM_011787 & Amfr & 2.38 \\
NM_023738 & Ube1l & 2.94 & NM_008948 & Psmc3 & 2.34 \\
NM_025770 & Apg10l & 2.92 & NM_008947 & Psmc1 & 2.29 \\
NM_023224 & Cblc & 2.91 & NM_019748 & Ublela & 2.26 \\
NM_021313 & Rnf25 & 2.82 & NM_010797 & Midl & 2.24 \\
NM_011909 & Usp18 & 2.82 & NM_027804 & Usp19 & 2.24 \\
NM_016786 & Hip2 & 2.78 & NM_015821 & Fbxl8 & 2.22 \\
NM_013907 & Fbxw4 & 2.68 & NM_145628 & Usp11 & 2.21 \\
NM_021522 & Usp14 & 2.65 & NM_015822 & Fbxl3a & 2.2 \\
NM_008950 & Psmc5 & 2.58 & NM_019803 & Ube2g2 & 2.19 \\
NM_018812 & Pias3 & 2.53 & NM_013890 & Fbxw2 & 2.17 \\
NM_010727 & Lnx1 & 2.53 & NM_021360 & Cul2 & 2.16 \\
NM_011543 & Skpla & 2.48 & NM_026557 & Zfp363 & 2.09 \\
NM_133247 & Usp33 & 2.43 & NM_009172 & Siah1a & 2.09 \\
NM_133777 & Ube2s & 2.43 & NM_008569 & Anapc1 & 2.05 \\
NM_009462 & Usp10 & 2.40 & NM_023137 & Ubd & 2.01 \\
\hline
\end{tabular}


Table 20. Down-regulated genes related to the ubiquitin-proteasome system in the mouse fetal liver following gestational Cd exposure.

\begin{tabular}{llcllc}
\hline Accession Number & Gene Name & Fold of change & Accession Number & Gene Name & Fold of change \\
\hline NM_080428 & Fbxw7 & 0.48 & NM_011664 & Ubb & 0.49 \\
NM_019639 & $U b c$ & 0.49 & & & \\
\hline
\end{tabular}

Table 21. Other up-regulated genes in the mouse fetal liver following gestational $\mathrm{Cd}$ exposure.

\begin{tabular}{|c|c|c|c|c|c|}
\hline Accession Number & Gene Name & Fold of change & Accession Number & Gene Name & Fold of change \\
\hline NM_019764 & Amotl2 & 3.58 & NM_007864 & $D \operatorname{lgh} 4$ & 2.40 \\
\hline NM_146279 & MOR224-12 & 3.55 & NM_019419 & Arl6ip1 & 2.40 \\
\hline NM_027022 & Cklfsf $2 a$ & 3.53 & NM_026826 & Mrps $18 \mathrm{c}$ & 2.39 \\
\hline NM_010167 & Eya4 & 3.48 & NM_010443 & Hтох2 & 2.39 \\
\hline NM_054080 & Akrlc20 & 3.48 & NM_008610 & Mmp2 & 2.39 \\
\hline NM_010863 & Myolb & 3.41 & NM_028639 & Ttc7 & 2.39 \\
\hline NM_025691 & $\operatorname{Srp} 72$ & 3.39 & NM_024182 & Riok3 & 2.39 \\
\hline NM_009278 & $S s b$ & 3.37 & NM_009378 & Thbd & 2.39 \\
\hline NM_021339 & Cdon & 3.35 & NM_007944 & Eps15-rs & 2.39 \\
\hline NM_017722 & Trmt1 & 3.32 & NM_008405 & Itgb2l & 2.39 \\
\hline NM_019924 & Rps6ka4 & 3.31 & NM_010833 & Msn & 2.39 \\
\hline NM_019837 & Nudt 3 & 3.30 & NM_053069 & Apg $5 l$ & 2.39 \\
\hline NM_080639 & Timp4 & 3.24 & NM_029355 & Prlpn & 2.39 \\
\hline NM_017389 & Ear4 & 3.23 & NM_013845 & Rorl & 2.39 \\
\hline NM_012045 & Pla 2 2f & 3.22 & NM_026309 & $L s m 3$ & 2.38 \\
\hline NM_016859 & Bysl & 3.20 & NM_011986 & $N c d n$ & 2.38 \\
\hline NM_023115 & Pcdh 15 & 3.20 & NM_008970 & Pthlh & 2.38 \\
\hline NM_018805 & $H s 3 s t 3 b$ & 3.20 & NM_013896 & Timm10 & 2.38 \\
\hline NM_025875 & $\operatorname{Rbm} 8$ & 3.19 & NM_008485 & Lamc2 & 2.38 \\
\hline NM_015836 & Wars2 & 3.18 & NM_029692 & Upp2 & 2.38 \\
\hline NM_009810 & Casp3 & 3.17 & NM_009746 & $B c l 7 c$ & 2.38 \\
\hline NM_007786 & Csnk & 3.17 & NM_007685 & $C f c l$ & 2.38 \\
\hline NM_027974 & Efhcl & 3.14 & NM_019578 & Extl1 & 2.38 \\
\hline NM_019428 & Rnasep2 & 3.14 & NM_008856 & Prkch & 2.38 \\
\hline NM_009621 & Adamts 1 & 3.14 & NM_007882 & $D s c 3$ & 2.38 \\
\hline NM_030691 & $\operatorname{Igs} f 6$ & 3.13 & NM_028930 & $T m c 5$ & 2.38 \\
\hline NM_008027 & Flot1 & 3.13 & NM_008301 & Hspa2 & 2.37 \\
\hline NM_021508 & Myoz1 & 3.12 & NM_001081274 & Pgd & 2.37 \\
\hline NM_008715 & $D d x 26$ & 3.11 & NM_027545 & Cwf19l2 & 2.37 \\
\hline NM_018755 & Pgcp & 3.11 & NM_016675 & Cldn2 & 2.37 \\
\hline NM_009052 & $\operatorname{Rex3}$ & 3.11 & NM_007403 & Adam 8 & 2.37 \\
\hline NM_025304 & Lcmt1 & 3.10 & NM_028153 & Eml2 & 2.37 \\
\hline NM_010829 & Msh3 & 3.10 & Y12713 & Erv4 & 2.37 \\
\hline NM_021504 & Nglyl & 3.08 & NM_031187 & Mcpt7 & 2.37 \\
\hline NM_010181 & $F b n 2$ & 3.08 & NM_013675 & Spnbl & 2.36 \\
\hline NM_016779 & Dmpl & 3.08 & NM_027726 & $D \lg 5$ & 2.36 \\
\hline NM_027513 & Nup205 & 3.07 & NM_138682 & Lrrc4 & 2.36 \\
\hline NM_010771 & Matr3 & 3.06 & NM_007734 & Col4a3 & 2.36 \\
\hline NM_016782 & Cntnap1 & 3.04 & NM_009177 & Siat4a & 2.36 \\
\hline NM_025597 & $N d u f b 3$ & 3.03 & NM_010084 & Adam18 & 2.36 \\
\hline NM_029810 & $N t 5 c 2$ & 3.03 & NM_028608 & Glipr1 & 2.36 \\
\hline NM_145535 & Sdcbp2 2 & 3.03 & NM_011851 & Nt5e & 2.36 \\
\hline
\end{tabular}


Gene expression changes in fetal liver of gestational Cd-exposed mice

Table 21. (Continued).

\begin{tabular}{|c|c|c|c|c|c|}
\hline Accession Number & Gene Name & Fold of change & Accession Number & Gene Name & Fold of change \\
\hline NM_025975 & Tctell & 3.02 & NM_008897 & Pon3 & 2.35 \\
\hline NM_030059 & Cst11 & 3.02 & NM_026768 & Mrps18a & 2.35 \\
\hline NM_007653 & Cd63 & 3.01 & NM_019800 & Acp6 & 2.35 \\
\hline NM_028860 & Mtmr3 & 3.01 & NM_007853 & Degs & 2.35 \\
\hline NM_009988 & Cxadr & 3.01 & NM_009729 & Atp6v0c & 2.35 \\
\hline NM_011500 & Strn & 3.00 & NM_011422 & Smrl & 2.35 \\
\hline NM_027181 & Pin4 & 3.00 & NM_130877 & Peg10 & 2.35 \\
\hline NM_026167 & Klhll3 & 2.99 & NM_011251 & Rbm6 & 2.35 \\
\hline NM_133167 & Parvb & 2.99 & NM_133726 & St13 & 2.35 \\
\hline NM_029916 & Stk31 & 2.99 & NM_033623 & Tes3 & 2.34 \\
\hline NM_008971 & Ptk9 & 2.99 & NM_011201 & Ptpn1 & 2.34 \\
\hline NM_028640 & Whrn & 2.98 & NM_027708 & Fbxo24 & 2.34 \\
\hline NM_021344 & Tesc & 2.98 & NM_009285 & Stcl & 2.34 \\
\hline NM_008579 & Meig1 & 2.98 & NM_010067 & Dnmt2 & 2.34 \\
\hline NM_013830 & $\operatorname{Prpf} 4 b$ & 2.98 & NM_007788 & Csnk2al & 2.34 \\
\hline NM_029492 & Zdhhc20 & 2.98 & NM_011657 & Tulp3 & 2.34 \\
\hline NM_019681 & Freq & 2.97 & NM_009121 & Sat1 & 2.34 \\
\hline NM_009356 & Tesp2 & 2.97 & NM_134048 & Cbll1 & 2.34 \\
\hline NM_011022 & $O t t$ & 2.96 & NM_001081306 & Ptprzl & 2.33 \\
\hline NM_033616 & Csprs & 2.96 & NM_025936 & Rars & 2.33 \\
\hline NM_018873 & $P 140$ & 2.95 & NM_020026 & B3galt3 & 2.33 \\
\hline NM_027926 & Cpa4 & 2.94 & NM_008980 & Ptpra & 2.33 \\
\hline NM_030719 & Gats & 2.94 & NM_021335 & Snrpb2 & 2.32 \\
\hline NM_010244 & $F v 1$ & 2.94 & NM_010837 & Mtap6 & 2.32 \\
\hline NM_008446 & Kif4 & 2.94 & NM_021292 & $E v c$ & 2.32 \\
\hline NM_013470 & Anxa3 & 2.93 & NM_029325 & Spinlw1 & 2.32 \\
\hline NM_008441 & Kiflb & 2.93 & NM_013774 & Tcllb4 & 2.31 \\
\hline NM_008403 & Itgblbpl & 2.92 & NM_008316 & Husl & 2.31 \\
\hline NM_026328 & $\operatorname{Reg} 4$ & 2.92 & NM_028454 & $\operatorname{Tm} 7 s f 2$ & 2.31 \\
\hline NM_020570 & $X r c c 2$ & 2.91 & NM_028493 & Rhobtb3 & 2.31 \\
\hline NM_028756 & Slc $35 a 5$ & 2.91 & NM_025441 & Sdccagl & 2.31 \\
\hline NM_011168 & Prlpf & 2.91 & NM_019870 & Ard1 & 2.31 \\
\hline NM_019637 & Styx & 2.91 & NM_019964 & Dnajb8 & 2.31 \\
\hline NM_008941 & $\operatorname{Prss} 7$ & 2.91 & NM_013759 & Sepr & 2.31 \\
\hline NM_008187 & Gtl3 & 2.91 & NM_145380 & Ga17 & 2.31 \\
\hline NM_007860 & Diol & 2.90 & NM_013848 & Ermap & 2.31 \\
\hline NM_011287 & Rpl10a & 2.90 & M11051 & $F v 4$ & 2.31 \\
\hline NM_033373 & $K r t 1-23$ & 2.90 & NM_032540 & $\mathrm{Kel}$ & 2.31 \\
\hline NM_015757 & Pcdhgal2 & 2.90 & NM_024449 & Sost & 2.30 \\
\hline NM_026969 & $\operatorname{Sec} 3111$ & 2.90 & NM_007791 & Csrp1 & 2.30 \\
\hline AF067063 & LOC380878 & 2.90 & NM_011690 & Vars2 & 2.30 \\
\hline NM_025768 & Grtp1 & 2.89 & NM_013771 & Yme1l1 & 2.30 \\
\hline NM_016713 & Map4k6 & 2.89 & NM_009639 & Crisp3 & 2.30 \\
\hline NM_008546 & Mfap2 & 2.88 & NM_031493 & $X 7 r 5$ & 2.30 \\
\hline NM_145371 & Eif $2 b 1$ & 2.88 & NM_021394 & Zbpl & 2.30 \\
\hline NM_019694 & Letm 1 & 2.88 & NM_016884 & Hnrpc & 2.30 \\
\hline NM_030744 & Ropn1 & 2.88 & NM_007683 & Cenpc & 2.30 \\
\hline NM_019519 & Rabggta & 2.87 & NM_017374 & Ppp2cb & 2.30 \\
\hline NM_009493 & $V 2 r 4$ & 2.87 & NM_023149 & $\mathrm{Cn} 2$ & 2.29 \\
\hline
\end{tabular}


H. Kurita et al.

Table 21. (Continued).

\begin{tabular}{|c|c|c|c|c|c|}
\hline Accession Number & Gene Name & Fold of change & Accession Number & Gene Name & Fold of change \\
\hline NM_010268 & Gdap10 & 2.87 & NM_134017 & Mat $2 b$ & 2.29 \\
\hline NM_019961 & Pex3 & 2.87 & NM_030711 & Arts1 & 2.29 \\
\hline NM_010795 & Mgat3 & 2.87 & NM_008662 & Myo6 & 2.29 \\
\hline NM_009049 & Resp18 & 2.87 & NM_207687 & Espn & 2.29 \\
\hline NM_029211 & Rnf121 & 2.87 & NM_001310623 & Wbp3 & 2.29 \\
\hline NM_145919 & Dorz1 & 2.87 & NM_027802 & Oboxl & 2.29 \\
\hline NM_010686 & Laptm 5 & 2.86 & NM_013664 & Sh3gll & 2.29 \\
\hline NM_008969 & $\operatorname{Ptgs} 1$ & 2.86 & NM_009377 & Th & 2.29 \\
\hline NM_025606 & Mrpl16 & 2.86 & NM_025407 & Uqcrcl & 2.28 \\
\hline NM_026871 & Hint2 & 2.85 & NM_010758 & Mag & 2.28 \\
\hline NM_025989 & $G p 2$ & 2.85 & NM_001174099 & Krt1-5 & 2.28 \\
\hline NM_029023 & Risc & 2.85 & NM_031874 & $R a b 3 d$ & 2.28 \\
\hline NM_008376 & Imap38 & 2.84 & NM_021286 & Sez6 & 2.28 \\
\hline NM_009420 & Crisp2 & 2.84 & NM_026483 & Mphosph10 & 2.28 \\
\hline NM_007688 & $C f 2$ & 2.84 & NM_025301 & Mrpl17 & 2.28 \\
\hline NM_008644 & Muc10 & 2.84 & NM_021360 & Neurl & 2.28 \\
\hline NM_009796 & Capn 7 & 2.84 & NM_019472 & Myo10 & 2.28 \\
\hline NM_007399 & Adam 10 & 2.84 & NM_011656 & Tuft1 & 2.28 \\
\hline NM_024187 & U2afl & 2.83 & NM_027879 & $C d c 40$ & 2.28 \\
\hline NM_025297 & Nrbfl & 2.83 & NM_007400 & Adam12 & 2.28 \\
\hline NM_009940 & $\operatorname{Coq} 7$ & 2.83 & NM_031257 & Plekha2 & 2.27 \\
\hline NM_031403 & Dbrl & 2.83 & NM_027777 & Pex1 & 2.27 \\
\hline NM_031256 & Plekha3 & 2.82 & NM_007415 & Adprt1 & 2.27 \\
\hline NM_026449 & Galnt15 & 2.82 & NM_019999 & Brp17 & 2.27 \\
\hline NM_015734 & Col5al & 2.81 & NM_008186 & Gtf $2 h 1$ & 2.27 \\
\hline NM_008625 & $M r c 1$ & 2.81 & NM_008586 & Meplb & 2.27 \\
\hline NM_053115 & Acox 2 & 2.80 & NM_011829 & $\operatorname{Impdh1}$ & 2.27 \\
\hline NM_009261 & Strbp & 2.80 & NM_080638 & $M v p$ & 2.26 \\
\hline NM_013456 & Actn3 & 2.80 & NM_145150 & Prcl & 2.26 \\
\hline NM_008770 & Cldn11 & 2.80 & NM_025369 & Mrps 36 & 2.26 \\
\hline NM_008868 & Pla2g2c & 2.80 & NM_021494 & Rab6ip1 & 2.26 \\
\hline NM_021898 & Halapx & 2.80 & NM_008939 & Prss 12 & 2.26 \\
\hline NM_030098 & Rnase6 & 2.79 & ВC003476 & $I i$ & 2.26 \\
\hline NM_148934 & Gtrgeo22 & 2.79 & NM_024241 & Kif 24 & 2.26 \\
\hline NM_016664 & Mata2 & 2.78 & NM_009867 & Cdh4 & 2.26 \\
\hline NM_013682 & $T 2$ & 2.78 & NM_013788 & Peg12 & 2.25 \\
\hline NM_023380 & Samsn 1 & 2.78 & NM_133673 & Tor $1 b$ & 2.25 \\
\hline NM_026329 & Polr2g & 2.78 & NM_009885 & $\mathrm{Cel}$ & 2.25 \\
\hline NM_013559 & Hsp105 & 2.78 & NM_021311 & Piwill & 2.25 \\
\hline NM_013799 & Atel & 2.78 & NM_015819 & Hs6st2 & 2.25 \\
\hline NM_011371 & Siat7a & 2.78 & NM_080454 & Gja12 & 2.25 \\
\hline NM_011452 & Serpinb9b & 2.77 & NM_011884 & Rngtt & 2.25 \\
\hline NM_009273 & Srp14 & 2.77 & NM_007862 & $D \operatorname{lgh} 1$ & 2.25 \\
\hline NM_019445 & Fmn2 & 2.77 & NM_018798 & Ubqln 2 & 2.24 \\
\hline NM_016744 & Pdela & 2.77 & NM_008110 & $G d f 9$ & 2.24 \\
\hline NM_009254 & Serpinb6a & 2.77 & NM_021537 & Stk25 & 2.24 \\
\hline NM_011061 & Padi4 & 2.77 & NM_008389 & $I p p$ & 2.24 \\
\hline NM_015785 & $Z p b p$ & 2.77 & NM_013712 & $\operatorname{Itg} b 1 b p 2$ & 2.24 \\
\hline NM_138646 & Hps4 & 2.77 & NM_172253 & Twistnb & 2.24 \\
\hline
\end{tabular}


Gene expression changes in fetal liver of gestational Cd-exposed mice

Table 21. (Continued).

\begin{tabular}{|c|c|c|c|c|c|}
\hline Accession Number & Gene Name & Fold of change & Accession Number & Gene Name & Fold of change \\
\hline NM_010942 & Nsgl & 2.76 & NM_013730 & Slamf1 & 2.24 \\
\hline NM_019991 & Prlpm & 2.76 & NM_053098 & Lmod2 & 2.24 \\
\hline NM_009432 & $T s h b$ & 2.75 & NM_009374 & $\operatorname{Tgm} 3$ & 2.24 \\
\hline NM_009015 & Rad54l & 2.75 & NM_009478 & Urod & 2.24 \\
\hline NM_013722 & Syn3 & 2.75 & NM_007647 & Entpd5 & 2.24 \\
\hline NM_010770 & Matn3 & 2.75 & NM_007690 & Chdl & 2.24 \\
\hline NM_009272 & Srm & 2.74 & NM_009351 & Tep1 & 2.24 \\
\hline NM_177369 & Myh8 & 2.74 & NM_016667 & Sntbl & 2.24 \\
\hline NM_027759 & Fsip1 & 2.74 & NM_028901 & Myolsb & 2.23 \\
\hline NM_025882 & Pole4 & 2.74 & NM_021525 & Rcll & 2.23 \\
\hline NM_021478 & Tulp1 & 2.74 & NM_016962 & Spg4 & 2.23 \\
\hline NM_019724 & Mmp16 & 2.74 & NM_010743 & Illrll & 2.23 \\
\hline NM_030710 & Ly108 & 2.74 & NM_009132 & Scin & 2.23 \\
\hline NM_133933 & Rpnl & 2.73 & NM_016753 & $L x n$ & 2.23 \\
\hline NM_009253 & Serpina $3 m$ & 2.73 & NM_025357 & Smpx & 2.23 \\
\hline NM_027874 & Csnkld & 2.73 & NM_011927 & Сeacam9 & 2.23 \\
\hline NM_019753 & Cdh17 & 2.73 & NM_138605 & Ppp1r3f & 2.22 \\
\hline NM_019765 & $R s n$ & 2.73 & NM_011375 & Siat9 & 2.22 \\
\hline NM_010904 & Nefh & 2.72 & NM_010085 & Adam 26 & 2.22 \\
\hline NM_133203 & Klra17 & 2.72 & NM_018736 & Mrella & 2.22 \\
\hline NM_020622 & ORF9 & 2.72 & NM_011517 & Sycp 3 & 2.22 \\
\hline NM_145381 & Lactb2 & 2.71 & NM_032006 & Mmpla & 2.22 \\
\hline NM_053161 & Mrpl27 & 2.71 & NM_023135 & Sult1e1 & 2.22 \\
\hline NM_008397 & Itga6 & 2.71 & NM_008497 & $L h b$ & 2.21 \\
\hline NM_013608 & $\mathrm{Naca}$ & 2.71 & NM_025448 & Ssr2 & 2.21 \\
\hline NM_028038 & $D d x 28$ & 2.71 & NM_023511 & Krtap3-1 & 2.21 \\
\hline NM_010027 & $D d t$ & 2.71 & NM_028099 & Dusp11 & 2.21 \\
\hline NM_019670 & Diap3 & 2.71 & NM_030717 & Lactb & 2.21 \\
\hline NM_027890 & Susd2 & 2.70 & NM_011240 & Ranbp 2 & 2.21 \\
\hline NM_010932 & Pnoc & 2.70 & NM_020046 & Dhodh & 2.21 \\
\hline NM_019461 & Usp27x & 2.70 & NM_022011 & $G t f 2 h 2$ & 2.21 \\
\hline NM_019425 & Gnpnat1 & 2.70 & NM_031376 & Pik3apl & 2.20 \\
\hline NM_024451 & Unc84a & 2.70 & NM_015769 & Ercc4 & 2.20 \\
\hline NM_029635 & Commd9 & 2.70 & NM_008035 & Folr2 & 2.20 \\
\hline NM_011341 & $S d f 4$ & 2.69 & NM_013679 & Svs6 & 2.20 \\
\hline NM_008880 & Plscr2 & 2.69 & NM_017397 & $D d x 20$ & 2.20 \\
\hline L24160 & Btps & 2.69 & NM_011910 & Uts2 & 2.20 \\
\hline NM_011074 & Pftk1 & 2.68 & NM_007992 & $F b \ln 2$ & 2.20 \\
\hline NM_021371 & Caln1 & 2.68 & NM_026716 & Sycn & 2.19 \\
\hline NR_004446 & $H 2-K 2$ & 2.68 & NM_009193 & Slbp & 2.19 \\
\hline NM_008102 & Gch & 2.68 & NM_008785 & Serpina5 & 2.19 \\
\hline NM_019834 & Git2 & 2.68 & NM_008547 & Mak & 2.19 \\
\hline NM_145705 & $\operatorname{Tinf} 2$ & 2.67 & AK009918 & $C d k 3$ & 2.19 \\
\hline NM_008525 & Alad & 2.67 & NM_177594 & Mtmr9 & 2.19 \\
\hline NM_031378 & Mlze & 2.67 & NM_008918 & Ppy & 2.19 \\
\hline NM_010917 & Nid1 & 2.67 & NM_009257 & Serpinb5 & 2.19 \\
\hline NM_133355 & Grid2ip & 2.67 & NM_007725 & Cnn2 & 2.19 \\
\hline NM_007697 & Chll & 2.67 & NM_018749 & Eif $3 s 7$ & 2.19 \\
\hline NM_020329 & Dolpp1 & 2.67 & NM_021489 & $F 12$ & 2.19 \\
\hline
\end{tabular}


H. Kurita et al.

Table 21. (Continued).

\begin{tabular}{|c|c|c|c|c|c|}
\hline Accession Number & Gene Name & Fold of change & Accession Number & Gene Name & Fold of change \\
\hline NM_011779 & Corolc & 2.67 & NM_010121 & Eif2ak3 & 2.18 \\
\hline NM_026314 & Dyxlc1 & 2.67 & NM_001285839 & Osgepl1 & 2.18 \\
\hline NM_013879 & Cabpl & 2.67 & NM_007645 & $C d 37$ & 2.18 \\
\hline NM_130457 & Cntnap4 & 2.67 & NM_025469 & Clps & 2.18 \\
\hline NM_026401 & Mrp63 & 2.67 & NM_009755 & Bmpl & 2.18 \\
\hline NM_021567 & Pcbp4 & 2.67 & NM_053090 & Drctnnbla & 2.18 \\
\hline NM_015755 & Hunk & 2.66 & NM_146007 & Colba2 & 2.18 \\
\hline NM_146087 & Csnklal & 2.66 & NM_029553 & $T t c 8$ & 2.18 \\
\hline NM_018753 & $Y w h a b$ & 2.66 & NM_017379 & Tubas & 2.18 \\
\hline NM_013882 & Gtse1 & 2.66 & NM_009419 & Tpst2 & 2.17 \\
\hline NM_020293 & Cldn9 & 2.66 & NM_007681 & Cenpa & 2.17 \\
\hline NM_015780 & Cfhll & 2.66 & NM_008277 & $H p d$ & 2.17 \\
\hline NM_008126 & Gjb3 & 2.65 & NM_009364 & Tfpi2 & 2.17 \\
\hline NM_027749 & Cpvl & 2.65 & ВC003885 & $M G C 6735$ & 2.17 \\
\hline NM_001005223 & Myohd1 & 2.65 & NM_021495 & Pvrl3 & 2.17 \\
\hline NM_007586 & Calb2 & 2.65 & NM_007745 & Cort & 2.17 \\
\hline NM_008958 & Ptch2 & 2.65 & NM_009645 & Aicda & 2.17 \\
\hline NM_019864 & Atr & 2.65 & NM_011796 & Capn10 & 2.17 \\
\hline NM_020330 & Adam 21 & 2.64 & NM_016978 & Oat & 2.17 \\
\hline NM_024193 & Nol5a & 2.64 & NM_011778 & Corolb & 2.17 \\
\hline NM_027276 & $C d c 16$ & 2.64 & NM_019410 & $P f n 2$ & 2.17 \\
\hline NM_052977 & Adarb2 & 2.64 & NM_133737 & Lancl2 & 2.17 \\
\hline NM_019576 & Thsd1 & 2.64 & NM_019684 & Stk23 & 2.17 \\
\hline NM_025450 & Mrps 17 & 2.64 & NM_008536 & $\operatorname{Tm} 4 s f 1$ & 2.17 \\
\hline NM_031997 & Tmem2 & 2.64 & NM_008412 & $I v l$ & 2.16 \\
\hline NM_009399 & Tnfrsfl1a & 2.64 & NM_027185 & Def6 & 2.16 \\
\hline NM_027100 & $R w d d 2$ & 2.64 & NM_007990 & Fau & 2.16 \\
\hline NM_029735 & Eprs & 2.64 & NM_020587 & Sfrs 4 & 2.16 \\
\hline NM_181073 & Plekhh1 & 2.64 & NM_008399 & Itgae & 2.16 \\
\hline NM_007919 & Ela2 & 2.63 & NM_010438 & $H k 1$ & 2.16 \\
\hline NM_178603 & Mrpl50 & 2.63 & NM_030562 & $\operatorname{Lrfn} 1$ & 2.16 \\
\hline NM_009947 & Cpne6 & 2.63 & NM_013714 & Irebfl & 2.16 \\
\hline NM_011166 & Prlpb & 2.63 & NM_008929 & Dnajc3 & 2.16 \\
\hline NM_009961 & Pcdhalo & 2.63 & NM_008634 & Mtaplb & 2.16 \\
\hline NM_011891 & Sgcd & 2.63 & NM_052994 & Spock2 & 2.15 \\
\hline NM_016712 & Tmod4 & 2.63 & NM_027892 & Ppp1r12a & 2.15 \\
\hline NM_030880 & Pacsin 3 & 2.62 & NM_053124 & Smarca5 & 2.15 \\
\hline NM_026033 & Odag & 2.62 & NM_020605 & $J p h 3$ & 2.15 \\
\hline NM_022565 & $N d s t 4$ & 2.62 & NM_009358 & Ppp2r5d & 2.15 \\
\hline NM_011729 & Ercc5 & 2.61 & NM_011992 & Rcn 2 & 2.15 \\
\hline NM_016740 & S100a11 & 2.61 & NM_021505 & Anapc5 & 2.15 \\
\hline NM_033572 & Wbscr 16 & 2.61 & NM_025294 & Gtlf3b & 2.15 \\
\hline NM_033612 & Ela1 & 2.61 & NM_009179 & Siat 5 & 2.15 \\
\hline AK008824 & $N u d t 7$ & 2.61 & NM_011561 & $T d g$ & 2.15 \\
\hline NM_008254 & $\mathrm{Hmgcl}$ & 2.60 & NM_029963 & Mrps 5 & 2.15 \\
\hline NM_009647 & $A k 4$ & 2.60 & NM_017468 & Enam & 2.15 \\
\hline NM_011995 & Pclo & 2.60 & NM_027351 & Ppil3 & 2.15 \\
\hline NM_016662 & Mad3 & 2.60 & NM_001025599 & Trim 26 & 2.15 \\
\hline NM_027570 & Ldhd & 2.60 & NM_020566 & Dnajc4 & 2.15 \\
\hline
\end{tabular}


Gene expression changes in fetal liver of gestational Cd-exposed mice

Table 21. (Continued).

\begin{tabular}{|c|c|c|c|c|c|}
\hline Accession Number & Gene Name & Fold of change & Accession Number & Gene Name & Fold of change \\
\hline NM_019636 & Tbcldl & 2.60 & NM_144800 & Mtss 1 & 2.14 \\
\hline NM_016963 & Tmod3 & 2.60 & NM_010238 & $\operatorname{Brd} 2$ & 2.14 \\
\hline NM_007730 & Coll2a1 & 2.60 & NM_008590 & Mest & 2.14 \\
\hline NM_013754 & Insl6 & 2.60 & NM_009310 & $P v r$ & 2.14 \\
\hline NM_027117 & Klhdc2 & 2.59 & NM_011906 & Tpra40 & 2.14 \\
\hline NM_010418 & Herc2 & 2.59 & NM_175337 & Mlh3 & 2.14 \\
\hline NM_027151 & Dctn2 & 2.59 & NM_025520 & $\operatorname{Lsm} 5$ & 2.14 \\
\hline NM_011285 & $R p g r$ & 2.58 & NM_007936 & Epha4 & 2.14 \\
\hline NM_009868 & Cdh5 & 2.58 & NM_026129 & Erp29 & 2.13 \\
\hline NM_009369 & $T g f b i$ & 2.57 & NM_011304 & Ruvbl2 & 2.13 \\
\hline NM_013747 & Golga 5 & 2.57 & NM_013760 & Dnajb9 & 2.13 \\
\hline NM_027907 & Agxt2l1 & 2.57 & NM_028782 & Prss 15 & 2.13 \\
\hline NM_031248 & Mapbpip & 2.57 & NM_007839 & Dhx $x 15$ & 2.13 \\
\hline NM_008081 & Galgt2 & 2.57 & NM_026425 & Nat5 & 2.13 \\
\hline NM_011840 & Map $2 k 5$ & 2.57 & NM_172451 & Galnt6 & 2.13 \\
\hline NM_030737 & $V 3 R 7$ & 2.57 & NM_008448 & Kif $5 b$ & 2.13 \\
\hline NM_019487 & Hebp 2 & 2.56 & NM_008696 & Map4k4 & 2.12 \\
\hline NM_007958 & Smarcad1 & 2.56 & NM_026171 & $N v l$ & 2.12 \\
\hline NM_011215 & Ptprn2 & 2.56 & NM_013763 & Tbl2 & 2.12 \\
\hline NM_018883 & Camkk1 & 2.56 & NM_009475 & Upa & 2.12 \\
\hline NM_013552 & $\mathrm{Hmmr}$ & 2.55 & NM_026154 & Mrpl10 & 2.12 \\
\hline NM_011776 & Zp3 & 2.55 & NM_007401 & Adam5 & 2.12 \\
\hline NM_026123 & Unc50 & 2.55 & NM_016924 & ORF5 & 2.12 \\
\hline NM_029157 & $S f 3 a 3$ & 2.55 & NM_009903 & Cldn4 & 2.12 \\
\hline NM_027294 & Cklfsf 8 & 2.55 & NM_027366 & Ly6g6e & 2.12 \\
\hline NM_019983 & Rabgef1 & 2.55 & NM_009443 & Tgoln1 & 2.12 \\
\hline NM_011035 & Pakl & 2.55 & NM_021347 & Gsdm & 2.12 \\
\hline NM_009515 & Was & 2.55 & M63245 & Alas 1 & 2.11 \\
\hline NM_009175 & St6gall & 2.55 & NM_031164 & $F 13 b$ & 2.11 \\
\hline NM_033268 & Actn2 & 2.55 & NM_013490 & Chk & 2.11 \\
\hline NM_054069 & Psbpcl & 2.55 & NM_023587 & Ptplb & 2.11 \\
\hline NM_033073 & Krt2-7 & 2.54 & NM_011284 & Rpa2 & 2.11 \\
\hline NM_031398 & Usmgl & 2.54 & NM_010631 & $K i f c 3$ & 2.11 \\
\hline NM_021278 & $\operatorname{Tmsb} 4 x$ & 2.54 & NM_019421 & $425018-1$ & 2.11 \\
\hline NM_011289 & Rpl27 & 2.54 & NM_008769 & Otc & 2.11 \\
\hline NM_025595 & Mrpl51 & 2.53 & NM_020508 & Brd4 & 2.10 \\
\hline NM_020498 & Ly6i & 2.53 & NM_010480 & Hspca & 2.10 \\
\hline NM_027982 & Ppp2cz & 2.53 & NM_025900 & Dek & 2.10 \\
\hline NM_009092 & Rps17 & 2.53 & NM_026102 & Daam 1 & 2.10 \\
\hline NM_025449 & Nicnl & 2.53 & NM_031181 & Siglecl1 & 2.10 \\
\hline NM_010330 & $E m b$ & 2.53 & NM_011579 & $T g t p$ & 2.10 \\
\hline NM_021331 & $G 6 p c-r s$ & 2.53 & NM_029271 & Mrpl32 & 2.10 \\
\hline NM_010058 & Dmwd & 2.52 & NM_027560 & Arrdc2 & 2.10 \\
\hline NM_001037756 & Brms1l & 2.52 & NM_009100 & Rptn & 2.10 \\
\hline NM_033078 & Klrk1 & 2.52 & NM_011858 & Odz4 & 2.10 \\
\hline NM_028785 & Dock8 & 2.52 & NM_011223 & $P x n$ & 2.10 \\
\hline NM_029967 & Adamtsll & 2.51 & NM_013821 & $H s d 3 b 6$ & 2.10 \\
\hline NM_010243 & Fut9 & 2.51 & NM_001033173 & Usp31 & 2.10 \\
\hline NM_145628 & Usp11 & 2.51 & NM_026384 & Dgat2 & 2.09 \\
\hline
\end{tabular}


H. Kurita et al.

Table 21. (Continued).

\begin{tabular}{|c|c|c|c|c|c|}
\hline Accession Number & Gene Name & Fold of change & Accession Number & Gene Name & Fold of change \\
\hline NM_013773 & $T c l 1 b 1$ & 2.51 & NM_008458 & Serpina3c & 2.09 \\
\hline NM_008294 & $H s d 3 b 4$ & 2.51 & NM_021432 & Nap1l5 & 2.09 \\
\hline NM_013638 & Prm3 & 2.50 & NM_007573 & Clqbp & 2.09 \\
\hline NM_010173 & Faah & 2.50 & NM_030703 & Cpn1 & 2.09 \\
\hline NM_021406 & Trem 1 & 2.50 & NM_010326 & Gplba & 2.09 \\
\hline NM_022814 & Polydom & 2.50 & NM_013925 & Adat1 & 2.09 \\
\hline NM_009598 & Ace & 2.50 & NM_031379 & Tktl1 & 2.09 \\
\hline NM_015776 & Mfap 5 & 2.50 & NM_027481 & $S f 4$ & 2.09 \\
\hline NM_007927 & Emd & 2.50 & NM_133854 & Snapap & 2.08 \\
\hline AK016135 & Etnk1 & 2.50 & NM_001271433 & Haghl & 2.08 \\
\hline NM_029977 & Polq & 2.50 & NM_027526 & Rasgefla & 2.08 \\
\hline NM_007916 & $D d x 19$ & 2.49 & NM_007733 & Col19al & 2.08 \\
\hline NM_010762 & Mal & 2.49 & NM_008474 & $K r t 2-16$ & 2.08 \\
\hline NM_025548 & Ckapl & 2.49 & NM_010221 & Fkbp10 & 2.08 \\
\hline NM_007744 & Comt & 2.49 & NM_010065 & Dnm & 2.08 \\
\hline NM_007800 & Ctsg & 2.49 & NM_009079 & Rpl22 & 2.08 \\
\hline NM_007933 & Eno3 & 2.49 & NM_024266 & Rps 25 & 2.08 \\
\hline NM_029791 & Bicd2 & 2.48 & NM_011782 & Adamts 5 & 2.07 \\
\hline NM_021385 & Rad18 & 2.48 & NM_007767 & Pcdha6 & 2.07 \\
\hline NM_010883 & $N d p h$ & 2.48 & NM_007391 & Acrvl & 2.07 \\
\hline NM_011651 & Stk22s 1 & 2.48 & NM_009038 & Rcvrn & 2.07 \\
\hline NM_026389 & Poldip2 & 2.47 & NM_028105 & Adckl & 2.07 \\
\hline NM_007841 & $D d x 6$ & 2.47 & NM_007738 & Col7al & 2.07 \\
\hline NM_008872 & Plat & 2.47 & NM_007926 & Scyel & 2.06 \\
\hline NM_016866 & Stk39 & 2.47 & NM_009613 & Adam 11 & 2.06 \\
\hline NM_007902 & $E d n 2$ & 2.47 & NM_009410 & Top3a & 2.06 \\
\hline NM_023210 & Anp32e & 2.46 & NM_013514 & Epb4.9 & 2.06 \\
\hline NM_008508 & Lor & 2.46 & NM_009130 & Scg3 & 2.06 \\
\hline NM_013742 & Cars & 2.46 & NM_007434 & Akt2 & 2.05 \\
\hline NM_010491 & Iapp & 2.46 & NM_134104 & $N d u f s 5$ & 2.05 \\
\hline NM_025718 & Dnase112 & 2.46 & NM_013750 & Phlda3 & 2.05 \\
\hline NM_013893 & Ingaprp & 2.45 & NM_011580 & Thbs 1 & 2.05 \\
\hline NM_029337 & Ep400 & 2.45 & NM_020564 & Sult5al & 2.05 \\
\hline NM_001098170 & Pcdh10 & 2.45 & NM_029360 & $\operatorname{Tm} 4 s f 5$ & 2.05 \\
\hline NM_008475 & $K r t 2-4$ & 2.45 & NM_008402 & $\operatorname{Itgav}$ & 2.05 \\
\hline NM_009483 & $U t x$ & 2.45 & NM_011855 & Odzl & 2.05 \\
\hline NM_019645 & Pkpl & 2.45 & NM_018765 & Wbp 4 & 2.04 \\
\hline NM_027681 & Gnpda2 & 2.45 & NM_029796 & $\operatorname{Lrg} 1$ & 2.04 \\
\hline NM_019517 & Bace2 & 2.44 & NM_013560 & Hspbl & 2.04 \\
\hline NM_053068 & Chracl & 2.44 & NM_011883 & Rnf13 & 2.04 \\
\hline NM_032544 & Gtpbp3 & 2.44 & NM_029815 & Bcasl & 2.04 \\
\hline NM_011917 & $X r n 2$ & 2.44 & NM_031843 & Dpp7 & 2.04 \\
\hline NM_007495 & Astn1 & 2.44 & NM_028477 & Plpcd & 2.03 \\
\hline NM_011693 & Vcam1 & 2.44 & NM_019871 & Amacl & 2.03 \\
\hline NM_026639 & Art4 & 2.44 & NM_009536 & Ywhae & 2.03 \\
\hline NM_011213 & Ptprf & 2.44 & NM_010910 & $\mathrm{Nefl}$ & 2.03 \\
\hline NM_011844 & Mgll & 2.43 & NM_010590 & $J u b$ & 2.03 \\
\hline NM_008100 & $G c g$ & 2.43 & NM_008613 & Mns1 & 2.03 \\
\hline NM_030689 & Nptxr & 2.43 & NM_013525 & Gas 5 & 2.03 \\
\hline
\end{tabular}


Gene expression changes in fetal liver of gestational Cd-exposed mice

Table 21. (Continued).

\begin{tabular}{|c|c|c|c|c|c|}
\hline Accession Number & Gene Name & Fold of change & Accession Number & Gene Name & Fold of change \\
\hline NM_028152 & Mms19l & 2.43 & NM_008408 & Itm 1 & 2.03 \\
\hline NM_009274 & Srpk2 & 2.43 & NM_023876 & Elp4 & 2.02 \\
\hline NM_009957 & Pcdha 7 & 2.43 & NM_021891 & Fignll & 2.02 \\
\hline NM_009614 & Adam 15 & 2.43 & NM_013706 & $C d 52$ & 2.02 \\
\hline NM_012053 & Rpl8 & 2.43 & NM_025878 & Mrps18b & 2.02 \\
\hline NM_019467 & Aifl & 2.42 & NM_026116 & Bbs2 & 2.02 \\
\hline NM_013600 & Msh5 & 2.42 & NM_016843 & Scalo & 2.02 \\
\hline NM_009705 & $\operatorname{Arg} 2$ & 2.42 & NM_145567 & Hibadh & 2.02 \\
\hline NM_019501 & Tprt & 2.42 & NM_010363 & Gstzl & 2.02 \\
\hline NM_011740 & Ywhaz & 2.42 & NM_020494 & $D d x 24$ & 2.01 \\
\hline NM_053103 & Entpd7 & 2.42 & NM_027135 & $\operatorname{Sec} 24 d$ & 2.01 \\
\hline NM_008061 & G6pc & 2.41 & NM_009960 & Pcdhall & 2.01 \\
\hline NM_011789 & $A p c 2$ & 2.41 & NM_021527 & $M k k s$ & 2.01 \\
\hline NM_008028 & Flot2 & 2.41 & NM_019744 & Ncoa4 & 2.01 \\
\hline NM_145516 & Plekhb2 & 2.41 & NM_007419 & Adrbl & 2.01 \\
\hline NM_023554 & Nol7 & 2.41 & NM_053192 & $U c c 1$ & 2.01 \\
\hline AF282275 & MOR171-24 & 2.41 & NM_019429 & Prss 16 & 2.01 \\
\hline NM_011120 & Plfr & 2.41 & NM_022989 & Arl6ip6 & 2.00 \\
\hline NM_008150 & Gpc4 & 2.40 & NM_008253 & Hmgb3 & 2.00 \\
\hline NM_027246 & Snrpf & 2.40 & NM_025540 & $\operatorname{Sln}$ & 2.00 \\
\hline NM_011987 & Pla2g10 & 2.40 & NM_028787 & Slc $35 f 5$ & 2.00 \\
\hline NM_011519 & $S d c 1$ & 2.40 & NM_028874 & Snx19 & 2.00 \\
\hline NM_011521 & $S d c 4$ & 2.40 & & & \\
\hline
\end{tabular}

Table 22. Other down-regulated genes in the mouse fetal liver following gestational Cd exposure.

\begin{tabular}{llllll}
\hline Accession Number & Gene Name & Fold of change & Accession Number & Gene Name & Fold of change \\
\hline NM_025336 & Chchd3 & 0.232 & NM_009413 & Tpd52l1 & 0.448 \\
NM_152821 & Purg & 0.247 & NM_145396 & Tbl3 & 0.449 \\
NM_030699 & Ntng1 & 0.273 & NM_011214 & Ptpru & 0.450 \\
NM_133724 & Cno & 0.275 & NM_021304 & Abhd1 & 0.456 \\
NR_001463 & Xist & 0.279 & NM_183296 & Krtap16-3 & 0.456 \\
NM_147095 & MOR32-1 & 0.282 & NM_009115 & S100b & 0.457 \\
NM_009933 & Col6a1 & 0.286 & NM_020296 & Rbms1 & 0.457 \\
NM_134240 & V1rh16 & 0.289 & NM_001081210 & Mrpl44 & 0.460 \\
NM_007801 & Ctsh & 0.307 & NM_007742 & Collal & 0.461 \\
NM_028216 & Psca & 0.333 & NM_010581 & Cd47 & 0.461 \\
NM_013643 & Ptpn5 & 0.334 & NM_145613 & C1qtnf5 & 0.461 \\
NM_011626 & Tparl & 0.337 & NM_025768 & Grtp1 & 0.462 \\
NM_029963 & Mrps5 & 0.363 & NM_023202 & Ndufa7 & 0.465 \\
NM_021433 & Stx6 & NM_023240 & Eef1d & 0.467 \\
NM_010915 & Ngfa & 0.364 & NM_021314 & Tacc2 & 0.469 \\
NM_023248 & Sbds & 0.370 & NM_033525 & Npnt & 0.471 \\
NM_011271 & Rnase1 & 0.376 & NM_030082 & Hist3h2ba & 0.472 \\
NM_028233 & Lrpprc & 0.377 & NM_009936 & Col9a3 & 0.474 \\
NM_138747 & Nol1 & 0.386 & NM_021422 & Dnaja4 & 0.474 \\
NM_145139 & Eif3s6ip & 0.392 & NM_008513 & Lrp5 & 0.475 \\
NM_009418 & Tpp2 & 0.405 & NM_011079 & Phkg & 0.477 \\
NM_007376 & Pzp & 0.406 & NM_029010 & Glb1l & 0.480
\end{tabular}


Table 22. (Continued).

\begin{tabular}{llcllc}
\hline Accession Number & Gene Name & Fold of change & Accession Number & Gene Name & Fold of change \\
\hline NM_144868 & Pcnxl3 & 0.412 & NM_019873 & Fkbpl & 0.481 \\
AY073109 & MOR229-1 & 0.414 & NM_011045 & Pcna & 0.482 \\
NM_013457 & Add1 & 0.414 & NM_011560 & Tcte3 & 0.482 \\
NM_010722 & Lmnb2 & 0.418 & NM_145226 & Oas3 & 0.485 \\
NM_010717 & Limk1 & 0.420 & NM_133734 & Wdr23 & 0.486 \\
NM_021296 & Grpel2 & 0.422 & NM_145575 & Cald1 & 0.490 \\
NM_023314 & Eif4el3 & 0.422 & NM_134158 & Igsf7 & 0.493 \\
NM_177345 & Mapkap1 & 0.425 & NM_010702 & Lect2 & 0.493 \\
NM_010683 & Lamc1 & 0.427 & NM_134230 & V1re11 & 0.494 \\
NM_007918 & Eif4ebp1 & 0.427 & NM_010485 & Elavl1 & 0.495 \\
NM_008836 & Phxr5 & 0.428 & NM_008299 & Dnajb3 & 0.496 \\
NM_025287 & Spop & 0.431 & NM_009291 & Stra6 & 0.496 \\
NM_021327 & Tnip1 & 0.436 & NM_011353 & Serf1 & 0.497 \\
NM_009932 & Col4a2 & 0.436 & NM_009304 & Syngr2 & 0.497 \\
NM_011664 & Ubb & 0.441 & NM_022722 & Dpys & 0.498 \\
NM_022980 & Dscrll2 & 0.441 & NM_148948 & Dicer1 & 0.500 \\
NM_021300 & Ehox & 0.445 & & &
\end{tabular}

\section{REFERENCES}

Lee, J.Y., Tokumoto, M., Fujiwara, Y. and Satoh, M. (2013): Gene expression analysis using DNA microarray in HK-2 human proximal tubular cells treated with cadmium. J. Toxicol. Sci., 38, 959-962.

Levin, A.A. and Miller, R.K. (1980): Fetal toxicity of cadmium in the rat: maternal vs. fetal injections. Teratology, 22, 1-5.

Nishijo, M., Nakagawa, H., Honda, R., et al. (2002): Effects of maternal exposure to cadmium on pregnancy outcome and breast milk. Occup. Environ. Med., 59, 394-396; discussion 397.

Nishijo, M., Tawara, K., Honda, R., Nakagawa, H., Tanebe, K. and Saito, S. (2004): Relationship between newborn size and mother's blood cadmium levels, Toyama, Japan. Arch. Environ. Health, 59, 22-25.
Satoh, M., Koyama, H., Kaji, T., Kito, H. and Tohyama, C. (2002): Perspectives on Cadmium Toxicity Research. The Tohoku Journal of Experimental Medicine, 196, 23-32.

Tokumoto, M., Lee, J.Y., Fujiwara, Y. and Satoh, M. (2013a): DNA microarray expression analysis of mouse kidney following cadmium exposure for 12 months. J. Toxicol. Sci., 38, 799-802.

Tokumoto, M., Ohtsu, T., Honda, A., Fujiwara, Y., Nagase, H. and Satoh, M. (2011): DNA microarray analysis of normal rat kidney epithelial cells treated with cadmium. J. Toxicol. Sci., 36, 127-129.

Tokumoto, M., Ohtsu, T., Imai, S., Honda, A., Nagase, H. and Satoh, M. (2013b): DNA microarray analysis of hepatic gene expression in mice exposed to cadmium for 30 days. J. Toxicol. Sci., 38, 155-157. 